Virginia Commonwealth University

VCU Scholars Compass

2013

\title{
Superlinear increase of photoluminescence with excitation intensity in Zn-doped GaN
}

Michael A. Reshchikov

Virginia Commonwealth University, mreshchi@vcu.edu

Anita J. Olsen

Virginia Commonwealth University

Marilyn F. Bishop

Virginia Commonwealth University

Tom McMullen

Virginia Commonwealth University

Follow this and additional works at: http://scholarscompass.vcu.edu/phys_pubs

Part of the Physics Commons

Reshchikov, M.A., Olsen, A.J., Bishop, M.F., et al. Superlinear increase of photoluminescence with excitation intensity in Zn-doped GaN. Physical Review B, 88, 075204 (2013). Copyright (C) 2013 American Physical Society.

\section{Downloaded from}

http://scholarscompass.vcu.edu/phys_pubs/49

This Article is brought to you for free and open access by the Dept. of Physics at VCU Scholars Compass. It has been accepted for inclusion in Physics Publications by an authorized administrator of VCU Scholars Compass. For more information, please contact libcompass@vcu.edu. 


\title{
Superlinear increase of photoluminescence with excitation intensity in $\mathrm{Zn}$-doped GaN
}

\author{
Michael A. Reshchikov* and Anita J. Olsen \\ Department of Physics, Virginia Commonwealth University, Richmond, Virginia 23284-2000, USA \\ Marilyn F. Bishop and Tom McMullen \\ Department of Physics, Virginia Commonwealth University, Richmond, Virginia 23284-2000, USA \\ and Center for the Study of Biological Complexity, Virginia Commonwealth University, Richmond, Virginia 23284-2000, USA
}

(Received 20 June 2013; published 13 August 2013)

\begin{abstract}
We have observed a superlinear increase of photoluminescence (PL) intensity in a narrow range of excitation intensities for Zn-doped GaN. The characteristic intensity at which the abrupt increase occurs increases with increasing temperature. This is unlike the usual observations for defects in semiconductors in which the PL intensity increases linearly with excitation intensity, saturating at high intensity because defects become saturated with photogenerated charge carriers. The observed phenomenon is attributed to a redirection of electron and hole flow from nonradiative centers at low excitation intensity to a recombination path via the $\mathrm{Zn}_{\mathrm{Ga}}$ acceptor at high excitation intensity. This is the same explanation responsible for the abrupt thermal quenching of PL reported earlier [Reshchikov et al., Phys. Rev. B 84, 075212 (2011).]
\end{abstract}

DOI: 10.1103/PhysRevB.88.075204

PACS number(s): 78.55.Cr, 71.55.Eq

\section{INTRODUCTION}

In a previous paper ${ }^{1}$ it was shown that, for high-resistivity gallium nitride $(\mathrm{GaN})$ doped with zinc, the photoluminescence (PL) intensity of the blue luminescence (BL) band drops by several orders of magnitude with increasing temperature over an interval of only a few Kelvins, and this occurs at temperatures between $100 \mathrm{~K}$ and $300 \mathrm{~K}$. In addition, it was shown that the crossover temperature at which this drop occurs changes as a function of excitation power density $P_{\mathrm{exc}}$ or electron-hole-pair generation rate $G$. The model that was used to explain this phenomenon included three types of point defects: the $\mathrm{Zn}_{\mathrm{Ga}}$ acceptor, the $\mathrm{O}_{\mathrm{N}}$ shallow donor, and an unknown nonradiative center $S$. In this paper, we study in depth the excitation intensity dependence, and we see that the increase of the PL intensity $I^{P L}$ is superlinear.

Photoluminescence from deep-level defects in semiconductors has been studied for many years and, in general, is well understood. ${ }^{2-5}$ Usually, the defect-related PL intensity $I^{P L}$ increases linearly with $G$ for a wide range of excitation intensities and tends to saturate at high $G$ when defects become saturated with photogenerated charge carriers. ${ }^{5-7}$ However, even as early as 1939, studies of PL from phosphors and photoconductors ( $\mathrm{ZnS}, \mathrm{ZnCdS}$, and $\mathrm{CdS}$ ) showed a superlinear increase in PL intensity with $G$ that was commonly described by a power law $I^{P L} \propto G^{m}$, with powers of $m$ exceeding 1 and sometimes $2 .^{8-15}$ An important feature of this unusual PL behavior was its tunability with temperature. Specifically, the region of the nonlinear increase of PL efficiency shifted to higher $G$ as temperature increased or as the concentration of PL "killer centers" (centers of nonradiative recombination) increased. ${ }^{10,11,14}$ After several attempts to fit this nonlinear behavior of the PL with empirical formulas, ${ }^{15,16}$ Klasens $^{17}$ suggested an explanation based on a two-center model. His approach was to use Duboc's method of considering all possible combinations of simplified solutions of rate equations. ${ }^{18}$ However, because little was known about semiconductors at that time, the model that he produced had donors close to the valence band and acceptors close to the conduction band. Nevertheless, viewed as an empirical formula, his model had the virtue of being able to reproduce the main features of the unusual PL behavior, namely, the superlinear increase of PL intensity in a certain region of $G$, with that superlinear increase shifting to higher $G$ with increasing temperature or with increasing concentration of nonradiative centers. Klasens's formulation correctly attributed the tunable superlinear rise of PL to the crossover of a system from a quasiequilibrium population of defect states, with the lower states in the band gap mostly filled with electrons and the upper states mostly empty at low $G$, to a population inversion, with more electrons at higher energy states than at lower energy states at high $G$. Unfortunately, the Klasens model was forgotten, possibly because of the unphysical energies of the acceptor and donor levels, and the basic ideas were not modified to account for more recent developments in the understanding of semiconductor defects. For instance, the superlinear tunable rise of PL intensity in the double heterostructures of InGaAsP/InP could not be satisfactorily explained, ${ }^{19,20}$ even though the PL behavior was very similar to the one predicted by Klasens. ${ }^{17}$

However, our recent discovery of the abrupt thermal quenching in $\mathrm{Zn}$-doped GaN shows many of the same features of the superlinear increase in PL intensity. We found that two centers were not sufficient to explain the data, since we are constrained by the knowledge of the approximate energies of the acceptors and shallow donors, as well as constraints on various other parameters. ${ }^{1}$ Because of these constraints, it became apparent that a deep donor, responsible for nonradiative recombination, was necessary. In the previous paper, we showed that the model with three types of defects could predict the temperature dependence, and in this paper we show that it also predicts the nonlinear dependence of PL intensity on excitation intensity in the temperature range corresponding to PL quenching.

The organization of the paper is as follows. In Secs. II and III, the experimental details and results are presented. In Sec. IV, the rate-equation model, developed earlier and used to explain the PL thermal quenching behavior in 
high-resistivity semiconductors, is employed to analyze the excitation intensity dependence of the PL intensity. In Sec. V, the effect of the exponential absorption of the excitation light on the calculations is considered. In Sec. VI, a comparison of the results of the rate equation model and the experimental data is discussed, including constraints on the parameters and the inadequacy of older models. The conclusions are given in Sec. VII.

\section{EXPERIMENT}

We have studied several $\mathrm{Zn}$-doped GaN layers grown by the hydride vapor phase epitaxy method on the $c$-plane sapphire. These samples were produced by the Ostendo GaN Lab (formerly TDI, Inc.) and had thicknesses between 3 and $10 \mu \mathrm{m}$. All the samples exhibited unusual PL quenching in which the BL band intensity dropped abruptly at a characteristic temperature $T^{*}$ that depended on the excitation intensity. ${ }^{1}$ From these samples we selected three samples for detailed study of the excitation intensity dependence of PL, as listed in Table I. In this table, the concentration of $\mathrm{Zn}$ atoms [ $\mathrm{Zn}$ ] is found from the secondary ion mass spectrometry (SIMS), and the concentrations of main defect species $\left(\mathrm{Zn}_{\mathrm{Ga}}\right.$ acceptor, shallow donor, and the nonradiative deep donor) are estimated from the fit of the temperature dependencies of PL intensity in this work and in Refs. 1 and 21.

Steady-state PL was excited with a continuous-wave He-Cd laser $(50 \mathrm{~mW}$, photon energy $3.81 \mathrm{eV})$, and the PL signal was dispersed by a 1200 rules $/ \mathrm{mm}$ grating in a $0.3-\mathrm{m}$ monochromator and detected by a cooled photomultiplier tube. The excitation power density $\left(P_{\text {exc }}\right)$ was varied from $2 \times 10^{-7}$ to $0.3 \mathrm{~W} / \mathrm{cm}^{2}$ by using neutral density filters and an unfocused laser beam with a diameter of $4 \mathrm{~mm}$. Levels of $P_{\text {exc }}$ up to $100 \mathrm{~W} / \mathrm{cm}^{2}$ were obtained by using a focused beam with a diameter of $0.1-0.2 \mathrm{~mm}$. These power densities are related to the generation rate $G$ of electron-hole pairs by $G=1.6 \times$ $10^{23} P_{\text {exc }}\left[\mathrm{W} / \mathrm{cm}^{2}\right] \mathrm{cm}^{-3} \mathrm{~s}^{-1}$. This was determined by dividing $P_{\text {exc }}$ by the energy of the excitation photons and multiplying by the absorption coefficient $\alpha=10^{5} \mathrm{~cm}^{-1}$. Therefore, the generation rates varied from $G=3.2 \times 10^{16} \mathrm{~cm}^{-3} \mathrm{~s}^{-1}$ to $1.6 \times 10^{25} \mathrm{~cm}^{-3} \mathrm{~s}^{-1}$.

A closed-cycle optical cryostat was employed for the temperature range of $10 \mathrm{~K}-330 \mathrm{~K}$. The PL spectra were corrected for the response of the optical system. The absolute PL quantum efficiency is defined by $\eta=I^{P L} / G$, where $I^{P L}$ is the integrated PL intensity from a particular defect (from $\mathrm{Zn}_{\mathrm{Ga}}$ in the case of the BL band), and $G$ is the concentration of electronhole pairs created by UV illumination per second in the same volume. To find $\eta$, we compared the integrated intensity of the BL band with the PL intensity obtained from the standard GaN

TABLE I. Characteristics of the GaN samples.

\begin{tabular}{lccrrr}
\hline \hline & Thickness & {$[\mathrm{Zn}]$} & \multicolumn{3}{c}{ Calculated concentrations $\left(\mathrm{cm}^{-3}\right)$} \\
\cline { 4 - 6 } Sample & $(\mu \mathrm{m})$ & $\left(\mathrm{cm}^{-3}\right)$ & $N_{A}$ & $N_{D}$ & \multicolumn{1}{c}{$N_{S}$} \\
\hline s452 & 2.9 & $2 \times 10^{19}$ & $1.6 \times 10^{18}$ & $5 \times 10^{17}$ & $1 \times 10^{18}$ \\
s560 & 5.0 & Unknown & $4.3 \times 10^{17}$ & $1.7 \times 10^{17}$ & $2.5 \times 10^{17}$ \\
ap269 & 6.6 & $2.5 \times 10^{18}$ & $3 \times 10^{17}$ & $1.3 \times 10^{17}$ & $1.5 \times 10^{17}$ \\
\hline \hline
\end{tabular}
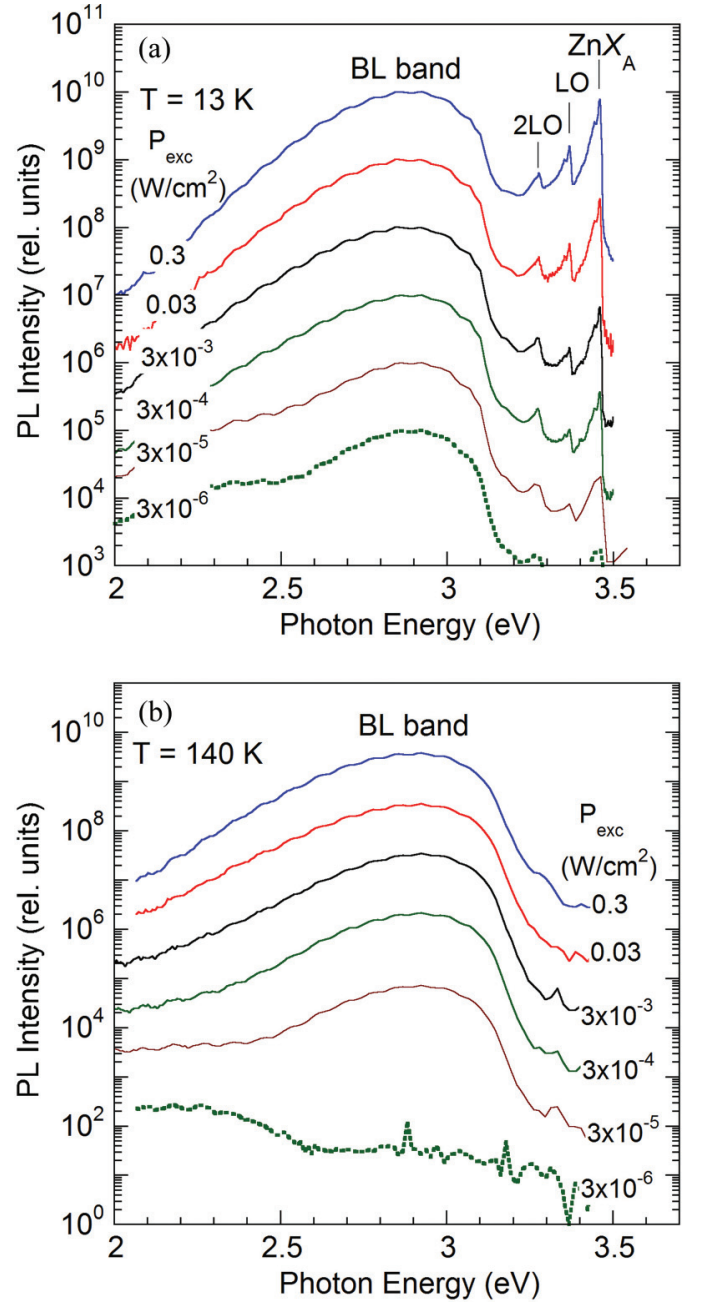

FIG. 1. (Color online) Photoluminescence spectrum of Zn-doped GaN (sample s452) for excitation power densities $P_{\text {exc }}$ between $3 \times$ $10^{-6}$ and $0.3 \mathrm{~W} / \mathrm{cm}^{2}$ with (a) $T=13 \mathrm{~K}$ and (b) $T=140 \mathrm{~K}$.

sample previously calibrated ${ }^{1}$ and measured under identical conditions and known excitation power density $P_{\text {exc }}$.

\section{RESULTS}

The low-temperature PL spectrum from a high-resistivity $\mathrm{GaN}: \mathrm{Zn}$ is shown in Fig. 1(a) for several excitation intensities. The peak at $3.458 \mathrm{eV}$ (labeled $\mathrm{Zn} X_{A}$ ) is attributed to an exciton bound to a neutral $\mathrm{Zn}_{\mathrm{Ga}}$ acceptor. This peak, followed by two LO phonon replicas, is the only exciton-related emission band in the near-band-edge region of the PL spectrum. The intensity of this band at $13 \mathrm{~K}$ increases with excitation intensity as a power law $\left(P_{\text {exc }}\right)^{m}$, where $m \approx 1.5$. In the defect-related region, the $\mathrm{BL}$ band with a maximum at $2.89 \mathrm{eV}$ is the dominant PL band. The BL band intensity increases linearly with excitation intensity at $13 \mathrm{~K}$. We observed similar intensity dependencies in other high-resistivity GaN:Zn samples.

With increasing temperature, the exciton emission quenches and almost disappears at $140 \mathrm{~K}$, as shown in Fig. 1(b). At this temperature, the BL band is slightly broader than at $13 \mathrm{~K}$ and has a maximum at $2.92 \mathrm{eV}$. It can be seen from Fig. 1(b) that at low excitation intensities (below $3 \times 10^{-5} \mathrm{~W} / \mathrm{cm}^{2}$ ) the BL band suddenly disappears. When 


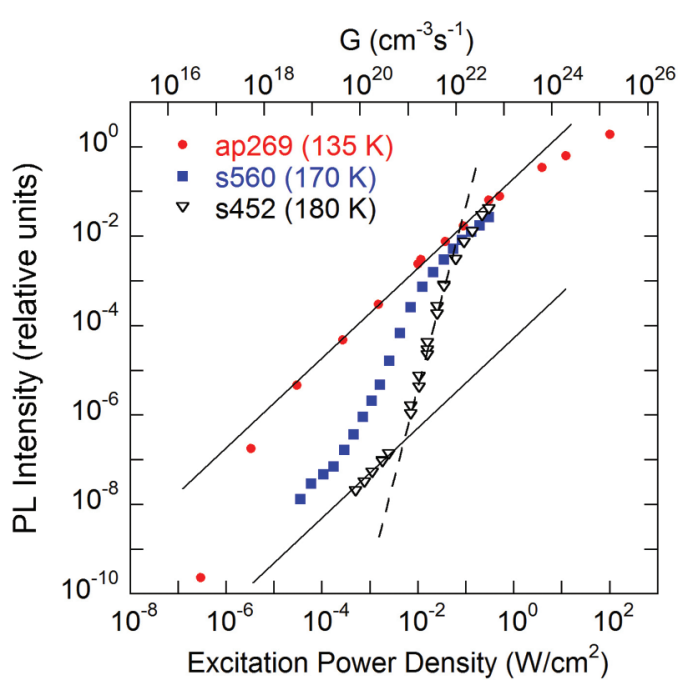

FIG. 2. (Color online) Dependence of PL intensity on excitation intensity for the BL band in three high-resistivity GaN:Zn samples at different temperatures. The solid lines indicate a linear dependence of the PL intensity on excitation intensity and the dashed line shows the highly nonlinear dependence, $I^{P L} \propto G^{m}$ with $m=4$.

the integrated PL intensity is plotted as a function of the excitation intensity, a superlinear increase is observed in a certain range, as shown in Fig. 2. Note that at lower and higher excitation intensities, a linear dependence was observed for the BL band. Such a nonlinear behavior in a narrow range of excitation intensity was observed for all high-resistivity $\mathrm{GaN}: \mathrm{Zn}$ samples. Figure 3 shows the dependence of PL

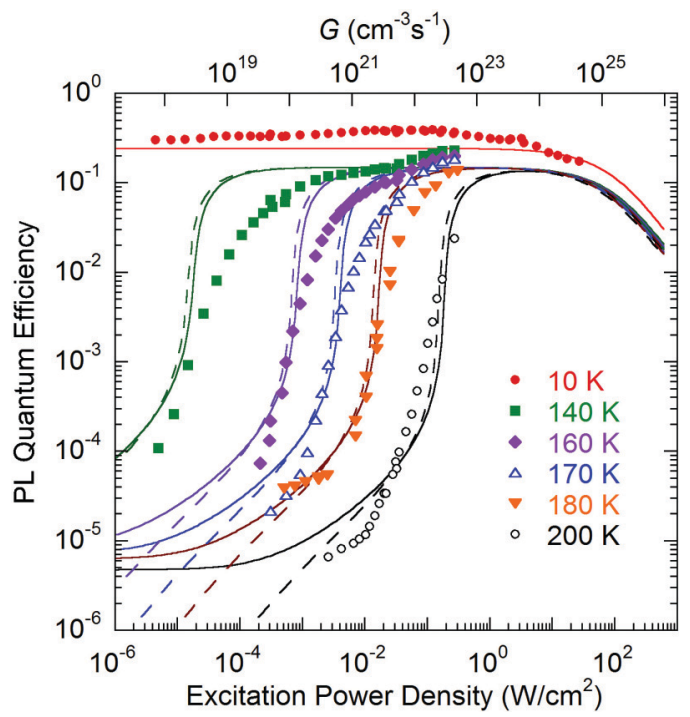

FIG. 3. (Color online) Dependence of the quantum efficiency of the $\mathrm{BL}$ band on excitation power density for selected temperatures for high-resistivity GaN:Zn (sample s452). The solid curves are for $p$ type and are calculated with the following parameters: $N_{A}=1.6 \times 10^{18} \mathrm{~cm}^{-3}, N_{S}=1 \times 10^{18} \mathrm{~cm}^{-3}, N_{D}=5 \times 10^{17} \mathrm{~cm}^{-3}$, $C_{n S}=2 \times 10^{-7} \mathrm{~cm}^{3} \mathrm{~s}^{-1}, C_{n D}=3 \times 10^{-8} \mathrm{~cm}^{3} \mathrm{~s}^{-1}, C_{p A}=7 \times$ $10^{-7} \mathrm{~cm}^{3} \mathrm{~s}^{-1}, C_{p S}=2 \times 10^{-6} \mathrm{~cm}^{3} \mathrm{~s}^{-1}, C_{n A}=4 \times 10^{-13} \mathrm{~cm}^{3} \mathrm{~s}^{-1}$, $C_{D A}=4 \times 10^{-12} \mathrm{~cm}^{3} \mathrm{~s}^{-1}, E_{D}=20 \mathrm{meV}, E_{A}=350 \mathrm{meV}, G=$ $1.6 \times 10^{23} \times P_{\text {exc }}\left[\mathrm{W} / \mathrm{cm}^{2}\right] \mathrm{cm}^{-3} \mathrm{~s}^{-1}$. The dashed curves are for $n$ type and are calculated with the same parameters except for $N_{A}=1.4 \times 10^{18} \mathrm{~cm}^{-3}$.

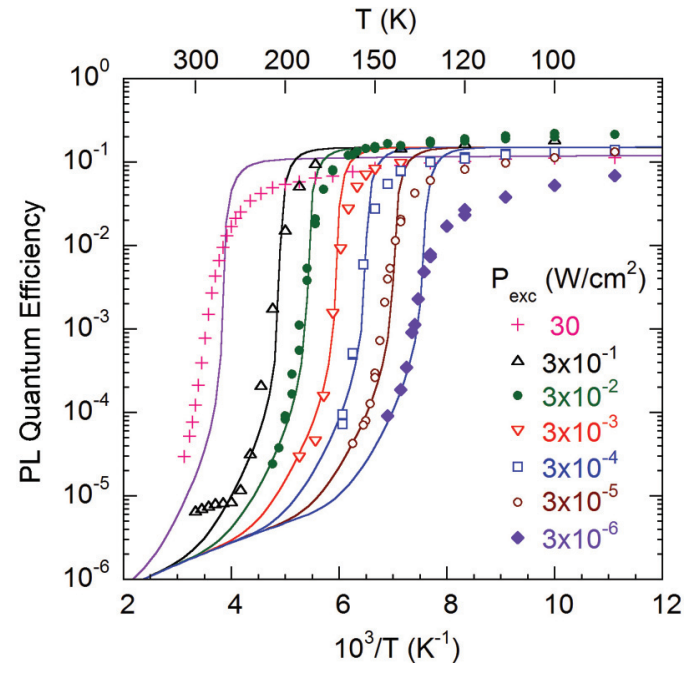

FIG. 4. (Color online) Temperature dependence of the quantum efficiency of the BL band for excitation power densities between $3 \times 10^{-6}$ and $30 \mathrm{~W} / \mathrm{cm}^{2}$ for high-resistivity GaN: $\mathrm{Zn}$ (sample s452). These correspond to $G$ ranging from $4.8 \times 10^{17} \mathrm{~cm}^{-3} \mathrm{~s}^{-1}$ to $4.8 \times 10^{24} \mathrm{~cm}^{-3} \mathrm{~s}^{-1}$. The solid curves are calculated with the same parameters as for Fig. 3 .

quantum efficiency on the excitation power density for one of the samples. The region of the abrupt, superlinear increase of the quantum efficiency of the BL band gradually shifts to higher excitation intensity with increasing temperature. The temperature dependence of the quantum efficiency of the BL band is shown in Fig. 4 for the same sample. The characteristic temperature, the temperature at which the PL intensity abruptly drops, gradually shifts from $130 \mathrm{~K}$ to $260 \mathrm{~K}$ with increasing excitation power density from $3 \times 10^{-6}$ to $30 \mathrm{~W} / \mathrm{cm}^{2}$. As demonstrated below, the nonlinear increase of PL intensity with increasing excitation intensity at fixed temperature, shown in Fig. 3, and the abrupt quenching of PL intensity with increasing temperature at fixed excitation intensity, as shown in Fig. 4, are two aspects of the same phenomenon.

\section{RATE-EQUATION MODEL}

The model presented in Ref. 1 successfully explained the temperature dependencies of PL in high-resistivity GaN:Zn, and it will be used here to explain the intensity dependencies. As shown in Fig. 5, the model includes three types of point defects: the $\mathrm{Zn}_{\mathrm{Ga}}$ acceptor, the $\mathrm{O}_{\mathrm{N}}$ shallow donor, and an unknown nonradiative deep donor $S$, which have total concentrations $N_{A}, N_{D}$, and $N_{S}$, respectively. The ionization energies for the acceptors and shallow donors are $E_{A}$ and $E_{D}$, respectively, while the $S$ center is sufficiently deep that thermal emission of charge carriers from it can be ignored. Two cases can be distinguished that result in nonlinear PL behavior in high-resistivity semiconductors: (1) an $n$-type semiconductor, for which $N_{D}+N_{S}>N_{A}>N_{D}$ and (2) a $p$-type semiconductor, for which $N_{A}>N_{D}+N_{S}$.

The rates of transitions that release energy are described with terms of the form $C_{\alpha \beta} N_{\alpha} N_{\beta}$, where the indices $\alpha$ and $\beta$ indicate the initial and final states of the transition, $N_{\alpha}$ and $N_{\beta}$ are the concentrations of the available carriers in the initial state and available empty sites in the final state, respectively, 


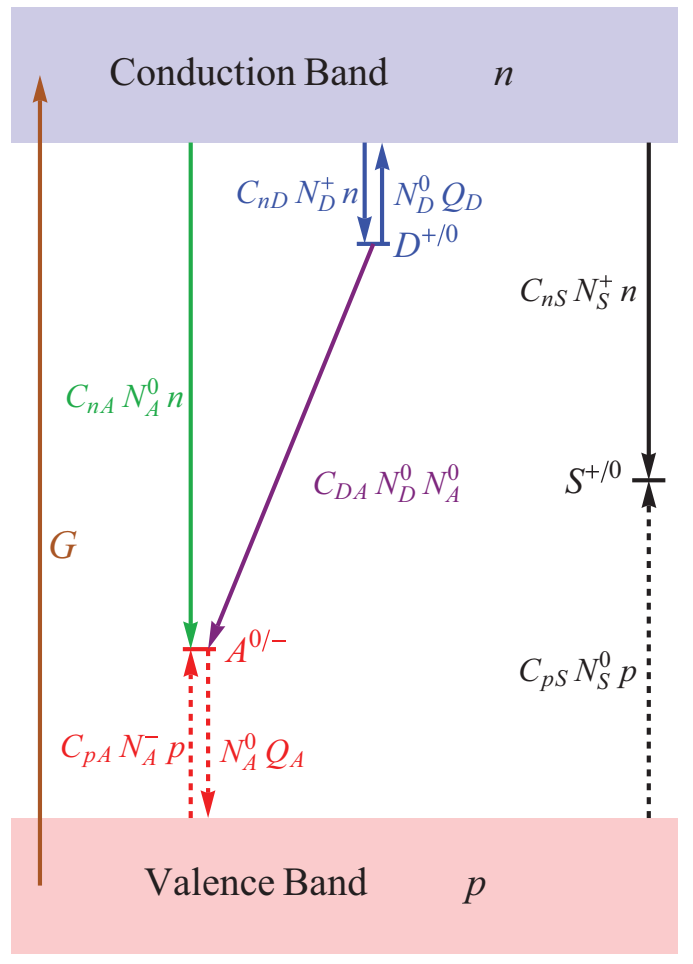

FIG. 5. (Color online) Band diagram and transitions of electrons (solid arrows) and holes (dashed arrows) in a semiconductor with a shallow donor $D$, acceptor $A$, and a nonradiative deep donor $S$.

and $C_{\alpha \beta}$ is the capture coefficient, assumed to be temperature independent for simplicity. The rates of thermal emission of electrons and holes from the defect levels to the conduction and valence bands are described by the emission coefficients

$$
\begin{aligned}
Q_{D} & =\frac{C_{n D} N_{c}}{g} \exp \left(-\frac{E_{D}}{k T}\right), \\
Q_{A} & =\frac{C_{p A} N_{v}}{g} \exp \left(-\frac{E_{A}}{k T}\right),
\end{aligned}
$$

respectively, where $N_{c}$ and $N_{v}$ are the effective densities of states in the conduction and valence bands, respectively, $k$ is Boltzmann's constant, and $g$ is the degeneracy factor of the donor and acceptor levels (assumed to be equal to 2 for both).

The rate equations under steady-state conditions can be written from the band diagram shown in Fig. 5 and are given by Eqs. (7)-(12) of Ref. 1 and are repeated in Eqs. (A1)-(A6) of the Appendix. The defect-related BL band in $\mathrm{GaN}: \mathrm{Zn}$ is caused by unresolved superposition of transitions of electrons from the conduction band and from the shallow donor level to the $\mathrm{Zn}_{\mathrm{Ga}}$ acceptor level; i.e., its intensity is

$$
I^{P L}=C_{D A} N_{D}^{0} N_{A}^{0}+C_{n A} n N_{A}^{0} .
$$

By solving the rate equations and the charge conservation equation explicitly given by Eqs. (A1)-(A6), we can determine how the PL intensity depends on excitation intensity at fixed temperature.

As we have established previously, ${ }^{1}$ the abrupt thermal quenching of PL separates two temperature regions divided by the solid red line in Fig. 6: a quasiequilibrium population of energy levels in the gap at high temperature to the left and below the red line (region I) and population inversion at low

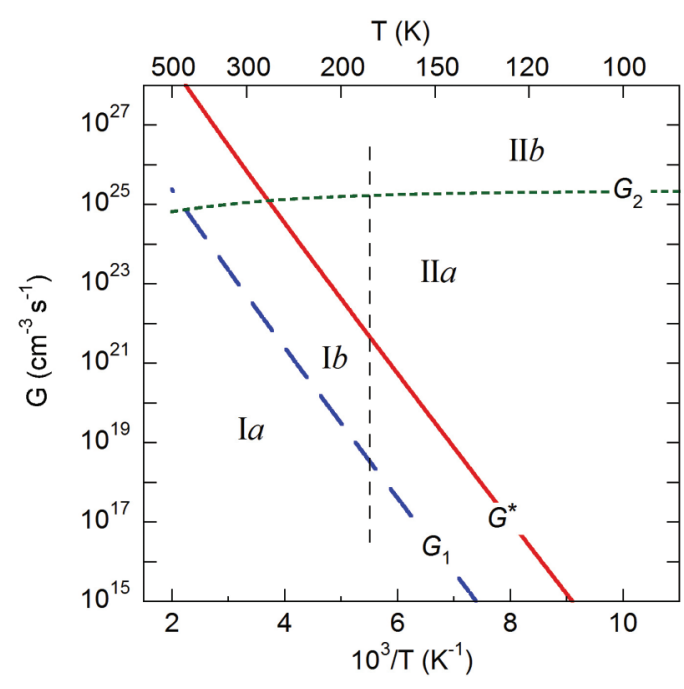

FIG. 6. (Color online) Characteristic regions for different PL behavior. The solid red line shows the $G^{*}(T)$ or $T^{*}(G)$ dependence. This line separates region I (to the left and below the line) from region II (to the right and above the line). The long-dashed blue line is $G_{1}(T)$, which separates subregions Ia and Ib, and the short-dashed green line is $G_{2}(T)$, which separates subregions IIa and IIb. For fixed $T$, the four regions (Ia, Ib, IIa, and IIb) analyzed in the current paper are indicated with the vertical dashed line at $T=180 \mathrm{~K}$. The parameters are the same as in Fig. 3.

temperature to the right and above the red line (region II). This line is characterized by the equation

$$
\ln G^{*}=\ln B-\frac{E_{A}}{k T},
$$

where

$$
B=C_{p A}\left(\frac{1}{\eta_{0}}-1\right)\left(N_{A}-N_{D}\right) \frac{N_{v}}{g}
$$

and

$$
\eta_{0}=\frac{C_{p A} N_{D}}{C_{p S} N_{S}+C_{p A} N_{D}} .
$$

Here $\eta_{0}$ is the constant quantum efficiency observed at high excitation intensity and low temperature, above the crossover, before it starts to decline at the highest intensities.

In Ref. 1, we focused on the temperature dependence for a given generation rate $G$, with the PL quenching abruptly at the solid line as one proceeds along a horizontal line in this figure. In that paper, the range of experimental generation rates $G$ never exceeded $10^{25} \mathrm{~cm}^{-3} \mathrm{~s}^{-1}$, and so the experimental data all lay in the regions shown as Ia, Ib, and IIa. Because the parameters shift the boundaries between regions, in this paper, some of the data fall in region IIb, as we will see in Sec. IV B. Here, in order to enable the reader to develop an intuitive idea of the processes involved, we proceed through the regions at a fixed temperature, starting in region I for low intensity and continuing to region II for high intensity. We then consider the narrow range of excitation intensities in which the crossover occurs.

In region I (low $G$ ), the majority of photogenerated electrons are quickly captured by the $S$ centers because they have a high electron-capture cross section. Since free holes 

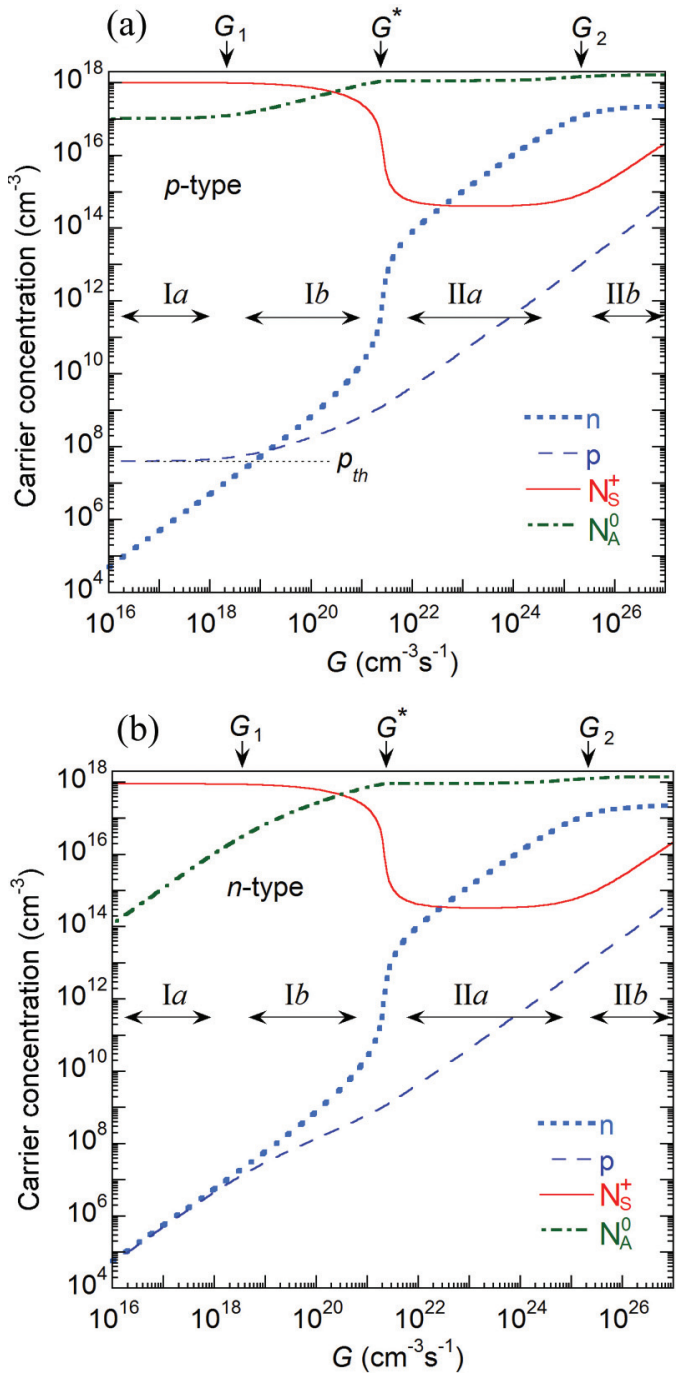

FIG. 7. (Color online) (a) $p$-type calculations and (b) n-type calculations of dependencies of concentrations of charge carriers on the electron-hole-pair generation rate $G$, where $G^{*}$ is the characteristic generation rate at which an abrupt crossover from low-efficiency to high-efficiency PL occurs. The curves are calculated with $T=180 \mathrm{~K}$ and with the other parameters the same as for Fig. 3.

are readily available due to their thermal emission from the $A$ center to the valence band, the electrons at $S$ centers recombine nonradiatively with these holes, leaving the $S$ centers empty of electrons for most of the time, so that for $p$ type, $N_{S}^{+} \approx N_{S}$. In region II (high $G$ ), due to the increasing rate of electron capture by $S$ centers and a lower rate of hole capture, the $S$ centers become saturated with photogenerated electrons, with $N_{S}^{+} \ll N_{S}$, and excess electrons accumulate in the conduction band. Moreover, the $A$ centers become saturated with holes because they capture holes much faster than they capture electrons. Thus, a population inversion is observed because the energy level of the $A$ center is lower than the energy level of the $S$ center. A crossover from region I to region II can be achieved either by variation of the temperature at a fixed excitation intensity (considered in detail in Ref. 1) or by variation of the excitation intensity at a fixed temperature. Below we consider how this transition takes place in $\mathrm{Zn}$-doped $\mathrm{GaN}$ at a fixed
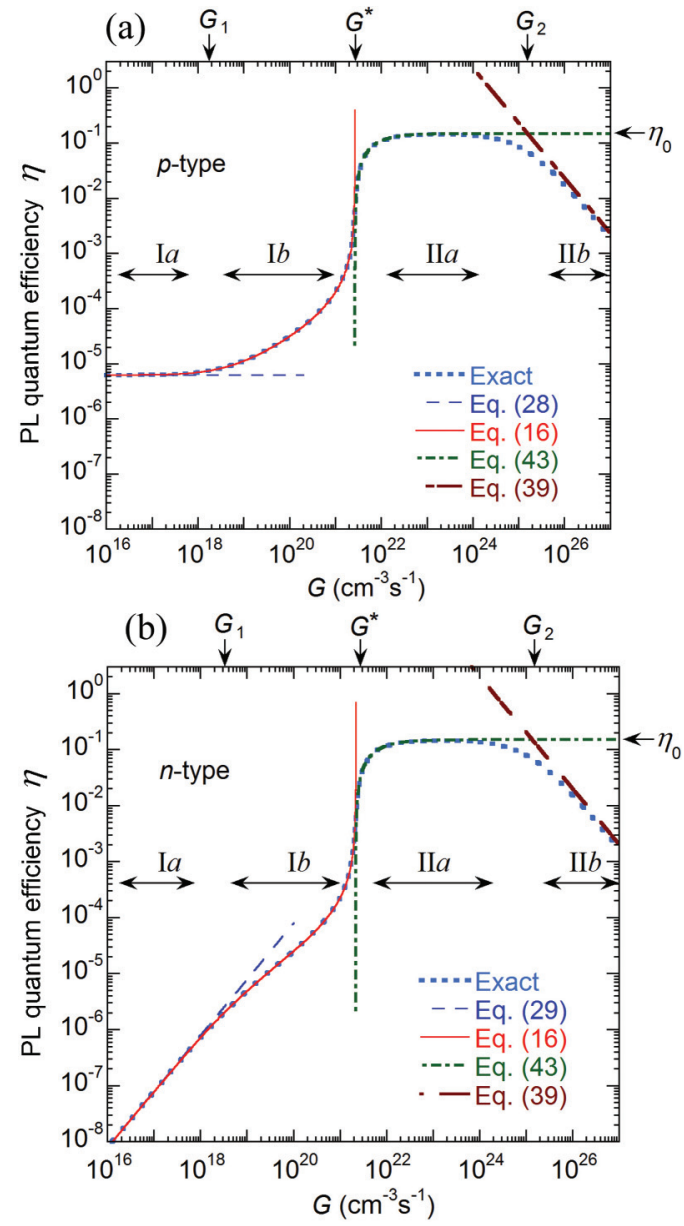

FIG. 8. (Color online) (a) $p$-type calculations and (b) $n$-type calculations of the dependence of the PL quantum efficiency on the electron-hole-pair generation rate $G$. Exact calculations were done using Eqs. (A1)-(A6) of the Appendix, and approximate calculations were done using Eqs. (16), (28), (29), (43), and (39). Model parameters are the same as for Fig. 3 with $T=180 \mathrm{~K}$.

temperature, and simple analytical expressions are derived for the different regions. The solutions with approximate analytical expressions will be compared to the numerical solutions of the rate equations, which are shown in Figs. 7 and 8 .

\section{A. Low excitation intensity}

At an excitation intensity below the crossover (region I, $\left.G<G^{*}\right)$, shallow donors $D$ are almost completely empty $\left(N_{D}^{+} \approx N_{D}\right)$. Because the excitation intensity is low, the concentrations of free electrons and holes are negligibly small, as can be seen in Fig. 7, and the charge conservation equation is simply

$$
N_{A}^{-}=N_{S}^{+}+N_{D}^{+} \approx N_{S}^{+}+N_{D}
$$

In this region, the free electrons $n$ in the conduction band are approximately in thermal equilibrium with the electrons $N_{D}^{0}$ bound to shallow donors, and so the rate of capture of electrons by shallow donors and the rate of thermal emission of electrons 
to the conduction band are almost equal; i.e.,

$$
C_{n D} N_{D}^{+} n \approx Q_{D} N_{D}^{0} \text {. }
$$

Moreover, in this region, the free holes $p$ in the valence band are approximately in thermal equilibrium with the acceptor states, and therefore the rate of capture of free holes by acceptors is nearly equal to the rate of thermal emission of holes to the valence band, so that

$$
C_{p A} N_{A}^{-} p \approx Q_{A} N_{A}^{0} .
$$

Also, the PL internal quantum efficiency $\eta$ is very low, since the capture coefficients $C_{D A}$ and $C_{n A}$ are small compared with $C_{n S}$ and $C_{p S}$, and as a consequence, the generated electron-hole pairs almost exclusively recombine through the nonradiative center $S$. Thus, the recombination rate at those centers is approximately equal to the generation rate $G$ of electron-hole pairs, and so we can write

$$
C_{n S} N_{S}^{+} n=C_{p S} N_{S}^{0} p \approx G .
$$

Combining Eqs. (7), (9), and (10), together with $N_{A}^{-}=N_{A}-$ $N_{A}^{0}$ and $N_{S}^{+}=N_{S}-N_{S}^{0}$, we obtain a quadratic equation for $N_{A}^{0}$, the solution of which is given by

$$
\begin{aligned}
N_{A}^{0}= & \frac{1}{2}\left(N_{A}-N_{S}-N_{D}-N_{2}\right) \\
& +\frac{1}{2} \sqrt{\left(N_{A}-N_{S}-N_{D}-N_{2}\right)^{2}+4 N_{A} N_{2},}
\end{aligned}
$$

where

$$
N_{2}=\frac{G C_{p A}}{Q_{A} C_{p S}} .
$$

This equation can also be found from the cubic equation for $N_{S}^{+}$from Eq. (B12) of Ref. 1 by setting $\zeta=0$, dividing out a factor of $N_{S}^{+}$, and substituting $N_{S}^{+}=N_{A}-N_{D}-N_{A}^{0}$. Here $\zeta$ is a small dimensionless parameter given by

$$
\zeta=\frac{C_{D A} C_{n D}}{C_{n S}} \frac{N_{D}}{Q_{D}}+\frac{C_{n A}}{C_{n S}} .
$$

Then $n$ can be found from Eq. (10) using Eq. (7), with $N_{A}^{-}=$ $N_{A}-N_{A}^{0}$,

$$
n \approx \frac{G}{C_{n S} N_{S}^{+}}=\frac{G}{C_{n S}\left(N_{A}-N_{D}-N_{A}^{0}\right)},
$$

and $N_{D}^{0}$ can be found from Eq. (8), where $N_{D}^{+} \approx N_{D}$, and is given by

$$
N_{D}^{0} \approx n \frac{C_{n D} N_{D}}{Q_{D}}
$$

The quantum efficiency $\eta_{\mathrm{I}}$ in region I can then be found from Eq. (3) as the PL intensity divided by $G$ as

$$
\eta_{\mathrm{I}}=\frac{I^{P L}}{G} \approx \frac{\zeta N_{A}^{0}}{N_{A}-N_{D}-N_{A}^{0}},
$$

where $N_{A}^{0}$ is given by Eq. (11) and $\zeta$ is given by Eq. (13). This expression is valid in all of region I ( $\mathrm{Ia}$ and $\mathrm{Ib}$ ).

For very low excitation intensity, or high temperature, corresponding to region Ia, $N_{2}$ becomes sufficiently small that it can be neglected in Eq. (11). Therefore, we define the dividing line in that equation as the point at which the sum of the terms inside the square root that are proportional to $N_{2}$ is equal to $\left(N_{A}-N_{D}-N_{S}\right)^{2}$. Then this characteristic generation rate $G_{1}$, which separates regions Ia and $\mathrm{Ib}$, is given by

$$
G_{1}=\frac{Q_{A} C_{p S}\left(N_{A}-N_{D}-N_{S}\right)^{2}}{2 C_{p A}\left(N_{A}+N_{D}+N_{S}\right)} .
$$

In the limiting case of region Ia, where $G \ll G_{1}$, the population of energy levels in the band gap is close to the equilibrium population in the dark and is different for a $p$-type than for an $n$-type semiconductor. Here we explain qualitatively how to envision this limiting case, and we present a rigorous derivation using a successive approximation method in the Appendix.

For $p$ type, $N_{A}>N_{D}+N_{S}$, and for $n$ type, $N_{A}<N_{D}+$ $N_{S}$. In Eq. (11), this determines whether the expression inside the square root nearly doubles or nearly cancels the first term in the expression. Expanding the square root, this means that for $p$ type,

$N_{A}^{0} \approx N_{A}-N_{D}-N_{S}+\Delta, \quad N_{A}^{-} \approx N_{D}+N_{S}-\Delta$,

where the small parameter $\Delta$ in the expansion is

$$
\Delta=\frac{N_{2} N_{A}}{\left(N_{A}-N_{D}-N_{S}\right)},
$$

with $N_{2}$ given in Eq. (12) and

$$
N_{S}^{+} \approx N_{S}-\Delta, \quad N_{S}^{0} \approx \Delta .
$$

Therefore, the $S$ centers are mostly ionized for $p$ type, while, for the numerical calculations of this paper, the $A$ centers are about $90 \%$ filled with electrons, i.e., $N_{A}^{-} \approx 0.9 N_{A}$.

For $n$ type,

$$
N_{A}^{-} \approx N_{A}+\Delta, \quad N_{A}^{0} \approx-\Delta,
$$

where $\Delta$ is negative, and

$$
N_{S}^{+} \approx N_{A}-N_{D}-\Delta, N_{S}^{0} \approx N_{D}+N_{S}-N_{A}+\Delta .
$$

Therefore, for $n$ type, the $A$ centers are almost completely filled with electrons, and for the numerical calculations of this paper, the $S$ centers are about $90 \%$ filled with holes; i.e., $N_{S}^{+} \approx 0.9 N_{S}$.

Finding an expression for the free hole concentration $p$ in region Ia is simplified by taking advantage of the quantities that are nearly constant for $p$ type and $n$ type. For $p$ type, it is the most convenient to use Eq. (9) since $N_{A}^{0}$ and $N_{A}^{-}$are nearly constant, and we obtain

$p \approx p_{\text {th }}=\frac{N_{A}^{0}}{N_{A}^{-}} \frac{Q_{A}}{C_{p A}}=\frac{\left(N_{A}-N_{D}-N_{S}\right)}{N_{D}+N_{S}} \frac{N_{v}}{g} \exp \left(-\frac{E_{A}}{k T}\right)$,

where we call $p_{t h}$ the thermally generated holes. For $n$ type, since $N_{S}^{0}$ is nearly constant, it is better to use Eq. (10), and we have

$$
p \approx p_{\text {opt }}=\frac{G}{C_{p S} N_{S}^{0}}=\frac{G}{C_{p S}\left(N_{D}+N_{S}-N_{A}\right)},
$$

where we call $p_{\text {opt }}$ the optically generated holes. At very low excitation intensity for $p$ type, since $p \approx p_{t h}$, the hole concentration is independent of $G$, as can be seen in Fig. 7(a) as the dark blue long-dashed curve or the horizontal black-dotted 


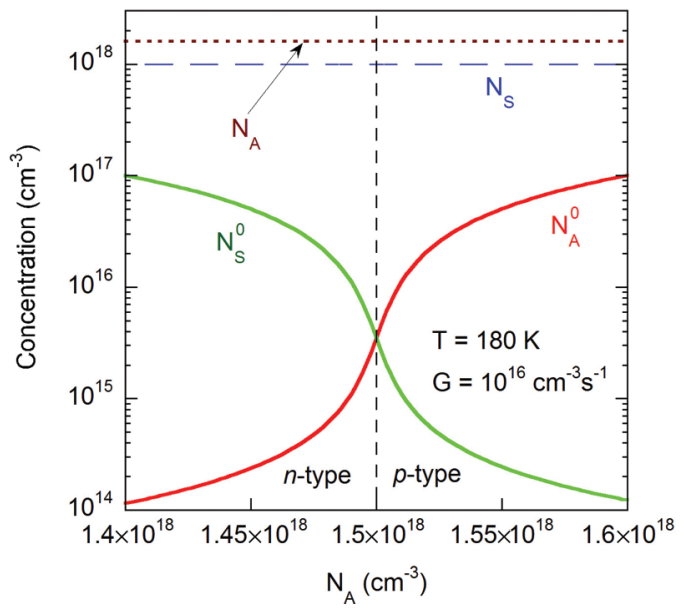

FIG. 9. (Color online) Neutral acceptor concentration $N_{A}^{0}$ and neutral deep donor concentration $N_{S}^{0}$ as a function of total acceptor concentration $N_{A}$. The parameters are the same as for Fig. 3 .

curve. For $n$ type, since $p \approx p_{\text {opt }}$, the hole concentration is primarily optically generated and increases linearly with $G$, as can be seen by the dark-blue long-dashed curve in Fig. 7(b). To illustrate why these results are different for $p$ type and $n$ type, we have plotted in Fig. 9 the concentrations $N_{A}^{0}$ and $N_{S}^{0}$ as a function of $N_{A}$. The middle of the graph, where $N_{A}=$ $N_{D}+N_{S}$, is for a completely compensated semiconductor, while to the left is $n$ type and to the right is $p$ type. We see from this figure that $N_{A}^{0}$ and $N_{S}^{0}$ trade places in $p$ type versus $n$ type, which, as we explain in the Appendix, is required by charge neutrality.

The concentration of free electrons in region Ia can now be obtained from Eq. (10) with $N_{S}^{+}=N_{S}$ for $p$ type as

$$
n \approx \frac{G}{C_{n S} N_{S}}
$$

and with $N_{S}^{+}=N_{A}-N_{D}$ for $n$ type as

$$
n \approx \frac{G}{C_{n S} N_{S}^{+}} \approx \frac{G}{C_{p S}\left(N_{A}-N_{D}\right)} .
$$

In both cases, $n$ increases linearly with $G$, as can be seen as the blue dotted curves in Figs. 7(a) and 7(b). From Eq. (8), we find the concentration of neutral shallow donors in terms of the free electron concentration as

$$
N_{D}^{0} \approx n \frac{C_{n D} N_{D}}{Q_{D}}=n \frac{g N_{D}}{N_{c}} \exp \left(\frac{E_{D}}{k T}\right) .
$$

The quantum efficiency for very low excitation intensity can be found from Eq. (16) and for $p$ type, with $N_{A}^{0}$ from Eq. (18), as

$$
\eta_{\mathrm{Ia}} \approx \zeta\left(\frac{N_{A}-N_{D}-N_{S}}{N_{S}}\right),
$$

which is a constant in region Ia, as can be seen in Fig. 8(a). In $n$ type, with $N_{A}^{0}$ from Eq. (21), the quantum efficiency can be written as

$$
\eta_{\mathrm{Ia}} \approx \frac{\zeta(-\Delta)}{\left(N_{A}-N_{D}\right)}=\frac{\zeta N_{A} N_{2}}{\left(N_{A}-N_{D}\right)\left(N_{D}+N_{S}-N_{A}\right)},
$$

where $N_{2}$ is defined in Eq. (12), and this increases linearly with $G$ in region Ia, as can be seen in Fig. 8(b). The fact that, in $p$ type, $\eta_{\mathrm{Ia}}$ is independent of $G$ implies that the PL intensity increases linearly with the excitation intensity. However, for $n$ type, $\eta_{\text {Ia }}$ is proportional to $G$ through $N_{2}$, and so the PL intensity for that case increases as $G^{2}$.

\section{B. High excitation intensity}

In the high excitation intensity region, region II $\left(G>G^{*}\right)$, analytical expressions all remain the same for the highresistivity $n$ type and the $p$ type materials because the concentration of optically generated holes exceeds the concentration of thermally generated holes. Due to the high capture rate $C_{n S}$ of electrons, the $S$ centers become saturated with electrons $\left(N_{S}^{0} \approx N_{S}\right)$ and are unable to act as efficient sinks for photogenerated conduction electrons. ${ }^{1}$ Instead, recombination occurs primarily through the radiative channel. Because of this, the rate of capture of holes by the $A$ center becomes much higher than the rate of thermal emission of bound holes to the valence band, and so the term $Q_{A} N_{A}^{0}$ can be neglected. As a consequence, the internal quantum efficiency of PL is much higher in this region than that in region I.

This means that the expression for the PL intensity related to the $A$ center can be found in terms of the free hole concentration by combining Eqs. (3) and (A5) as

$$
I^{P L} \approx C_{p A} N_{A}^{-} p
$$

where we have neglected the term $Q_{A} N_{A}^{0}$. Alternatively, the expression for the quantum efficiency can be written in terms of the free electron concentration, since the expression for $N_{D}^{0}$ given by Eq. (15) is still valid in this region. Using Eq. (3) and substituting for $n$ in terms of $N_{D}^{0}$ from Eq. (15), we have

$$
\begin{aligned}
I^{P L} & =\left(C_{D A} N_{D}^{0}+C_{n A} n\right) N_{A}^{0} \\
& \approx\left(\frac{C_{D A} C_{n D} N_{D}}{Q_{D}}+C_{n A}\right) n N_{A}^{0} \approx \zeta C_{n S} n N_{A}^{0},
\end{aligned}
$$

where $\zeta$ is given by Eq. (13).

Region IIa is defined for generation rates $G$ that are above the crossover $G^{*}$ but below a temperature-independent upper limit $G_{2}$, which will be determined later. In this region, the concentrations of free electrons $n$ and holes $p$ are still small, as can be seen in Fig. 7, and can be ignored in the charge neutrality equation, as was done for region I. Approximate analytical expressions for the quantum efficiency $\eta$ and the concentrations of free and bound electrons and holes can be found for this case because here $N_{D}^{0} \ll N_{D}$, as was true in region I, and here, in addition $N_{S}^{+} \ll N_{S}$. Consequently, the charge conservation equation reduces to

$$
N_{A}^{-} \approx N_{D}
$$

The concentrations of free holes and electrons in this region are proportional to $G$, as can be seen in Fig. 7. In particular, $p$ can be found from the rates of hole capture by both the acceptor and the deep donor through the equation

$$
G=C_{p A} N_{A}^{-} p+C_{p S} N_{S}^{0} p .
$$


Then substituting for $N_{A}^{-}$from Eq. (32), the concentration of free holes can be written from Eq. (33) as

$$
p \approx p_{0}=\frac{G}{C_{p S} N_{S}+C_{p A} N_{D}},
$$

where $p_{0}$ means optically generated, and $n$ can be found from Eqs. (30), (31), and (32) as

$$
n \approx \frac{C_{p A} N_{A}^{-} p_{0}}{\zeta C_{n S} N_{A}^{0}}=\frac{C_{p A} N_{D}}{\zeta C_{n S}\left(N_{A}-N_{D}\right)} p_{0} .
$$

As can be seen in Fig. 7, in this region $n \gg p$ for high-resistivity GaN:Zn because $\zeta \ll 1$, while $C_{n S}$ and $N_{D}$ are not much different from $C_{p A}$ and $N_{A}-N_{D}$, respectively. The quantum efficiency $\eta_{\text {IIa }}$ in this region is independent of $G$ because $N_{S}^{0} \approx N_{S}$ and assumes the form

$$
\eta_{\text {IIa }} \approx \eta_{0}=\frac{C_{p A} N_{D}}{C_{p S} N_{S}+C_{p A} N_{D}},
$$

and this constant quantum efficiency in region IIa is clearly seen in Fig. 8.

At a very high excitation intensity, region $\operatorname{IIb}\left(G>G_{2}\right)$, the acceptors become saturated with photogenerated holes. Therefore, in this region $N_{A}^{-} \ll N_{A}$ and $N_{S}^{+} \ll N_{S}$, as is clear from Fig. 7. The electron concentration increases greatly so that in this region the charge conservation equation becomes

$$
n \approx N_{D}^{+} \text {. }
$$

Then in Eq. (A3) of the Appendix, we set $N_{A}^{0}=N_{A}, N_{D}^{+}=n$ and $N_{D}^{0}=N_{D}-n$, which produces a quadratic equation in $n$. Also, since $C_{D A} N_{A} \ll Q_{D}$ for $T>100 \mathrm{~K}$, we neglect terms containing $C_{D A}$, and, solving the resulting quadratic equation, we obtain

$$
n=\frac{\sqrt{Q_{D}^{2}+4 Q_{D} C_{n D} N_{D}}-Q_{D}}{2 C_{n D}} .
$$

Then from Eqs. (3) and (37), we obtain an expression for the PL quantum efficiency in region IIb as

$$
\eta_{\text {IIb }}=\frac{\left[C_{D A}\left(N_{D}-n\right)+C_{n A} n\right] N_{A}}{G},
$$

where $n$ is given by Eq. (38). We can see from Eq. (39) that $\eta \propto G^{-1}$, as can be seen in Fig. 8, and the PL intensity therefore becomes independent of the excitation intensity.

By equating the quantum efficiencies in regions IIa and IIb given by Eqs. (36) and (39), respectively, we can now obtain an expression for the characteristic generation rate $G_{2}$ that separates regions IIa and $\mathrm{IIb}$ and is given by

$$
G_{2}=\frac{\left[C_{D A}\left(N_{D}-n\right)+C_{n A} n\right] N_{A}}{\eta_{0}},
$$

where $\eta_{0}$ is given by Eq. (36). A condition that is commonly satisfied for GaN:Zn samples is $C_{n D} N_{D} / Q_{D} \ll 1$ for $T>$ $100 \mathrm{~K}$. When that is true, by looking at Eq. (38), it can be seen that $n \ll N_{D}$. In this case, Eq. (40) can be simplified to

$$
\begin{aligned}
G_{2} & \approx \frac{N_{D} N_{A}}{\eta_{0}}\left[C_{D A}-\left(C_{D A}-C_{n A}\right) \sqrt{\frac{Q_{D}}{C_{n D} N_{D}}}\right] \\
& \approx \frac{C_{D A} N_{D} N_{A}}{\eta_{0}} .
\end{aligned}
$$

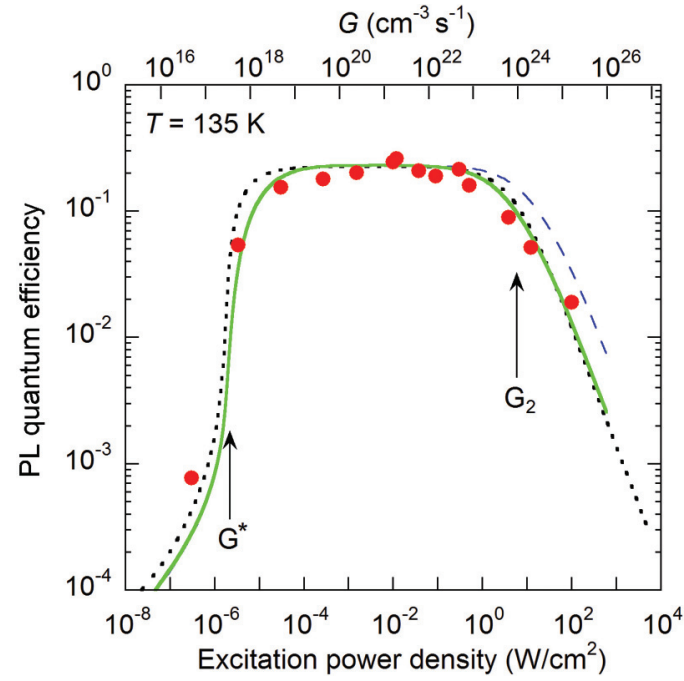

FIG. 10. (Color online) Dependence of the PL quantum efficiency on excitation power density for temperature $T=135 \mathrm{~K}$. The points are experimental data for sample ap269. The curves are the numerical solution of Eqs. (A1)-(A6) of the Appendix, calculated with the following parameters: $N_{S}=1.5 \times 10^{17} \mathrm{~cm}^{-3}, N_{A}=3 \times 10^{17} \mathrm{~cm}^{-3}$, $N_{D}=1.3 \times 10^{17} \mathrm{~cm}^{-3}, C_{n D}=10^{-8} \mathrm{~cm}^{3} \mathrm{~s}^{-1}, C_{p A}=10^{-6} \mathrm{~cm}^{3} \mathrm{~s}^{-1}$, $C_{p S}=3 \times 10^{-6} \mathrm{~cm}^{3} \mathrm{~s}^{-1}, C_{n A}=4 \times 10^{-13} \mathrm{~cm}^{3} \mathrm{~s}^{-1}, E_{D}=30 \mathrm{meV}$, $E_{A}=340 \mathrm{meV}, G=1.6 \times 10^{23} \times P_{\text {exc }}\left[\mathrm{W} / \mathrm{cm}^{2}\right] \mathrm{cm}^{-3} \mathrm{~s}^{-1}$. The dotted curve is calculated using the step-function absorption model and the long-dashed curve using the exponential absorption model with $\alpha=10^{5} \mathrm{~cm}^{-1}$, both using $C_{n S}=1 \times 10^{-7}$ and $C_{D A}=8 \times 10^{-12} \mathrm{~cm}^{3} \mathrm{~s}^{-1}$. The solid curve is calculated using the exponential absorption model with $C_{n S}=3 \times 10^{-8}$ and $C_{D A}=$ $2.4 \times 10^{-12} \mathrm{~cm}^{3} \mathrm{~s}^{-1}$.

For the parameters given in the caption to Fig. 3 with $T=$ $180 \mathrm{~K}$, we find that $G_{2}=1.2 \times 10^{25} \mathrm{~cm}^{-3} \mathrm{~s}^{-1}$ when it is calculated with Eq. (40) and $G_{2}=2.1 \times 10^{25} \mathrm{~cm}^{-3} \mathrm{~s}^{-1}$ when it is calculated with Eq. (41). The highest intensity shown in Fig. 4 is for $P_{\text {exc }}=30 \mathrm{~W} / \mathrm{cm}^{2}$, corresponding to $G=4.8 \times$ $10^{24} \mathrm{~cm}^{-3} \mathrm{~s}^{-1}$, which is still in region IIa. However, for sample ap269, $G_{2}=8.3 \times 10^{23}$ and $1.4 \times 10^{24} \mathrm{~cm}^{-3} \mathrm{~s}^{-1}$ when it is calculated with Eqs. (40) and (41), respectively. From this, we expect that the quantum efficiency will decrease for $P_{\text {exc }}$ above about $5 \mathrm{~W} / \mathrm{cm}^{2}$. This is illustrated in Fig. 10, where the experimental quantum efficiency, shown by the points, rises abruptly at $P_{\text {exc }}=10^{-6} \mathrm{~W} / \mathrm{cm}^{2}$, becomes constant for the range $10^{-5} \mathrm{~W} / \mathrm{cm}^{2}<P_{\text {exc }}<0.1 \mathrm{~W} / \mathrm{cm}^{2}$, and decreases for $P_{\text {exc }}>1 \mathrm{~W} / \mathrm{cm}^{2}$.

\section{Near the crossover $G^{*}(T)$}

The electron-hole-pair generation rate $G^{*}$ at which the system abruptly crosses over from region I to region II is given by Eq. (5), and at that point the PL efficiency $\eta$ jumps by orders of magnitude. In $p$ type, the ratio of the efficiency in region IIa divided by that in region Ia can be used as a measure of the size of the jump, since both of these are approximately constant. This ratio $R^{*}$, found by dividing Eq. (36) by Eq. (28), is given by

$$
R^{*} \approx \frac{\eta_{0} N_{S}}{\zeta\left(N_{A}-N_{D}-N_{S}\right)},
$$


where $\zeta$ is given by Eq. (13). This is the same ratio obtained on either side of the crossover as a function of temperature as given by Eq. (27) of Ref. 1. On the high-intensity side of the crossover, as $G$ approaches $G^{*}$, the PL quantum efficiency decreases with decreasing $G$, as given in Eqs. (20) and (B28) of Ref. 1, and varies as

$$
\eta=\eta_{0}\left[1-\frac{B}{G} \exp \left(-\frac{E_{A}}{k T}\right)\right] .
$$

Very close to the crossover, the quantum efficiency has the form given by Eq. (B31) of Ref. 1, which is

$$
\eta_{\text {cross }} \approx \zeta\left(\frac{N_{A}-N_{D}}{N_{S}^{+}}\right),
$$

and the slope on the log-log plot increases dramatically compared with the slopes on either side of the crossover. The slope right at the crossover is equivalent to finding the power $m$ in the expression $\eta \propto G^{m}$ and can be found by taking the logarithmic derivative of $\eta_{\text {cross }}$, given by

$$
m=\left.\frac{d \ln \eta_{\text {cross }}}{d \ln G}\right|_{G=G^{*}}=-\left.\frac{G}{N_{S}^{+}} \frac{d N_{S}^{+}}{d G}\right|_{G=G^{*}} .
$$

The concentration of positively charged deep donors very close to the crossover is given by Eq. (B30) of Ref. 1 and can be written as

$$
N_{S}^{+} \approx-\frac{b}{2 a}+\sqrt{-\frac{c}{a}},
$$

where $a, b$, and $c$ are the coefficients of a cubic polynomial in Eq. (B12) of Ref. 1, which gives results that are indistinguishable from the numerical solution. The coefficients are defined in Eqs. (B13)-(B15) of that paper. The slope is so steep because the coefficient $b$ decreases by several orders of magnitude right at the crossover compared with its values immediately to either side. The changes of the other coefficients are practically zero by comparison, and so, to a very good approximation, we can take only the derivative of the $b$ dependence. Also, $b$ is a function of $G$ only through its dependence on $N_{2}$ given in Eq. (12). Then the slope can be written as

$$
m=\left.\frac{C_{p A} G}{2 a N_{S}^{+} C_{p S} Q_{A}} \frac{d b}{d N_{2}}\right|_{G=G^{*}} .
$$

At the crossover, $d b / d N_{2} \approx N_{D}$, and $N_{S}^{+}$is dominated by the second term in Eq. (46), and

$$
\left.a N_{S}^{+}\right|_{G=G^{*}} \approx N_{S}\left(N_{A}-N_{D}\right) \sqrt{\zeta \lambda}
$$

where $\zeta$ is given by Eq. (13) and

$$
\lambda=\left(\frac{N_{S}}{N_{D}} \frac{C_{p S}}{C_{p A}}+1\right)\left(\frac{N_{A}}{N_{D}}+\frac{N_{A}-N_{D}}{N_{S}}\right) .
$$

The slope is then given by

$$
m=\frac{C_{p A} N_{D} G^{*}}{2 Q_{A} C_{p S} N_{S}\left(N_{A}-N_{D}\right) \sqrt{\zeta \lambda}} .
$$

For the parameters given in the caption to Fig. 3 and for $T=180 \mathrm{~K}$, we find that $G^{*}=2.7 \times 10^{21} \mathrm{~cm}^{-3} \mathrm{~s}^{-1}, \lambda=7.3$, $\zeta=6.3 \times 10^{-5}$, and the power of $G$ in the quantum efficiency dependence is $m=23.4$, a huge increase above the usual linear $(m=1)$ behavior.

\section{DEPTH DEPENDENCE OF THE INCIDENT LIGHT}

In the preceding discussion, the electron-hole excitation rate $G$ has been pictured as constant within an active layer of thickness $\sim 0.1 \mu \mathrm{m}$, which we call the step-function absorption model. In reality, the incident light is exponentially attenuated with a penetration depth of this order. This variation in the pair-generation rate $G$ with depth causes some smearing of the sharp features in the solution to the rate-equation model, and so these features would be less pronounced in the experimentally observed variation in the PL intensity with the excitation power. To get an idea of how important this attenuation is for the experimental data presented here, we construct a simple model, which we call the exponential absorption model, in which the generation rate $G$ varies with depth $x$ below the surface as $\exp (-\alpha x)$, where $\alpha$ is the absorption coefficient for $\mathrm{GaN}$ at $325 \mathrm{~nm}$, the wavelength of the incident light. In this model, the quantum efficiency is averaged over the thickness of the sample weighted by the depth-dependent generation rate $G(x)$ as

$$
\langle\eta\rangle=\frac{\int_{0}^{\infty} \eta(G(x)) G(x) d x}{\int_{0}^{\infty} G(x) d x},
$$

where

$$
G(x)=G(0) e^{-\alpha x},
$$

and $\alpha \approx 10^{5} \mathrm{~cm}^{-1}$. The upper limit on the integral is taken to be infinite because for GaN samples with thickness greater than $3 \mu \mathrm{m}$, the light intensity decays by more than 10 orders of magnitude before it reaches the back surface. For $G(x)$ constant over a depth $\alpha^{-1}$, this expression reduces to the stepfunction absorption model. The quantity that is plotted on the upper horizontal axis in Fig. 11 is the average of $G(x)$ within a layer of thickness $\alpha^{-1}$,

$$
\langle G(x)\rangle=\alpha \int_{0}^{\infty} G(x) d x=G(0),
$$

which is the value of the generation rate at the surface.

In order to see how this averaging of the quantum efficiency affects the sharp increase in quantum efficiency as a function of $\langle G(x)\rangle$, we first consider the extreme case of a step function $\eta(G)$ that is zero below the generation rate $G=G^{*}$ at which the abrupt increase occurs, and a constant value $\eta_{0}$ above,

$$
\eta(G)=\eta_{0} \Theta\left(G-G^{*}\right)
$$

where $\Theta\left(G-G^{*}\right)=1$ for $G>G^{*}$ and zero otherwise. To perform the integration in Eq. (51), it is necessary to find the depth $x^{*}$ at which $G=G^{*}$ using Eq. (52), yielding

$$
x^{*}=\frac{1}{\alpha} \ln \left(\frac{G(0)}{G^{*}}\right)
$$

for $G(0)>G^{*}$ and zero otherwise, because for $G(0)<G^{*}$ the integral in the numerator of Eq. (51) would be zero. Therefore, the average quantum efficiency becomes

$$
\langle\eta\rangle=\left(1-\frac{G^{*}}{G(0)}\right) \eta_{0} \Theta\left(G(0)-G^{*}\right) .
$$

In the inset to Fig. 11, the black dashed curve is the original step function for $\eta(x)$, which is what one would get with the step-function absorption model (with the initial flat region 


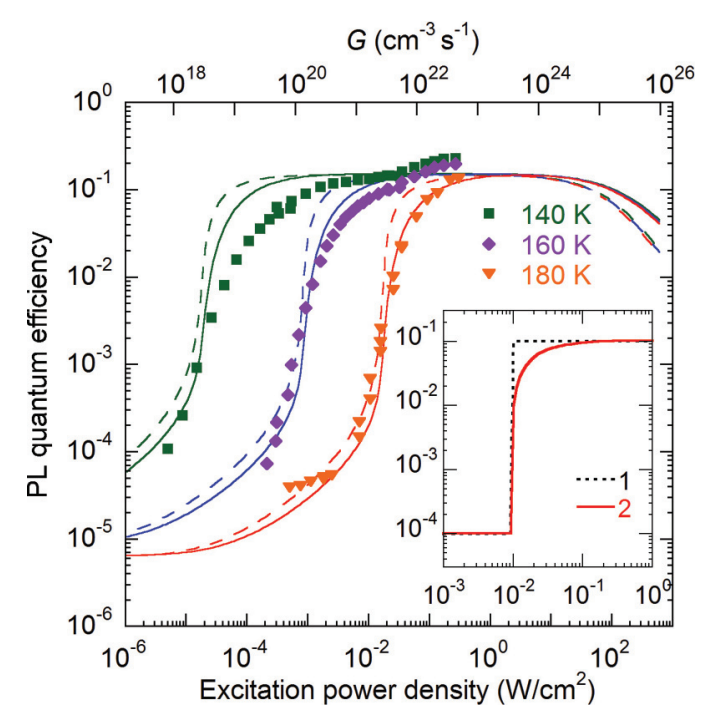

FIG. 11. (Color online) Dependence of the PL quantum efficiency on excitation power density for temperatures $T=140,160$, and $180 \mathrm{~K}$. Points are experimental data from sample s452. Dashed curves are the numerical solution of Eqs. (A1)-(A6) of the Appendix, with the assumption the excitation intensity does not change in the effective depth equal to the attenuation depth $\alpha^{-1}$. Solid curves are calculated with the same parameters using the exponential attenuation model with $\alpha=10^{5} \mathrm{~cm}^{-1}$. The parameters are the same as for Fig. 3 . The inset shows a hypothetical step function to mimic the quantum efficiency in the step-function absorption model (dashed curve 1) and its transformation using the exponential model (curve 2).

adjusted to be $10^{-4}$ instead of zero for purposes of illustration), and the red solid curve is $\langle\eta\rangle$ averaged over the depth in the exponential absorption model. It is clear that this averaging process rounds the shoulder of the curve because the crossover value of $G(x)=G^{*}$ at depth $x$ requires that the $G$ at the surface be higher. The same procedure has been used to calculate the effect of the depth dependence on the rate equation model discussed in Sec. IV, as shown in Fig. 11. The dashed curves are calculated with the step-function absorption model, using the numerical solution to Eqs. (A1)-(A6) of the Appendix with the parameters of Fig. 3, and the solid curves are calculated with the exponential absorption model using the same numerical solution. Clearly, the inclusion of the depth dependence of the generation rate improves the fit to the data.

We found parameters in Ref. 1 that fit the temperature dependencies of the quantum efficiency of the BL band for the GaN:Zn sample ap269 using the step-function absorption model, and we have used those same parameters to compare with the experimental data as a function of $G$. From the fit of these dependencies in the range of $P_{\text {exc }}$ from $2 \times 10^{-7} \mathrm{~W} / \mathrm{cm}^{2}$ to $0.3 \mathrm{~W} / \mathrm{cm}^{2}$ with Eqs. (7)-(12) of Ref. 1, we obtained the set of parameters for the model discussed in Sec. IV that includes three types of defects. In Fig. 10, we used these parameters to model the dependence of the BL band quantum efficiency on excitation power density for this sample using the step-function absorption model, and this result is shown as the dotted curve. When we used the same parameters with the exponential absorption model, we obtained the long-dashed curve, which is shifted to higher $G$ compared with the experimental data, where the left-hand part of this curve is the same as the solid curve. The solid curve is obtained by keeping the ratio $C_{D A} / C_{n S}$ fixed and decreasing both constants by a factor of 3 in order to fit the data. Keeping the ratio fixed is required to keep the left-hand part of the curve the same. Such a change in $C_{n S}$ and $C_{D A}$ does not affect the calculated temperature dependencies reported in Ref. 1 because the data in that paper did not extend into region IIb.

\section{DISCUSSION}

We now discuss the constraints on the model parameters that are imposed by analyzing both the temperature and excitation intensity dependencies. Dependencies of the PL quantum efficiency on excitation intensity were fit with numerical solutions of Eqs. (A1)-(A6) of the Appendix, and, as can be seen in Fig. 3, the fit reproduces the main features of the experimental data. For instance, at $10 \mathrm{~K}$, the quantum efficiency is independent of excitation intensity over a wide range and starts decreasing above $10 \mathrm{~W} / \mathrm{cm}^{2}$. For temperatures between $140 \mathrm{~K}$ and $200 \mathrm{~K}$, the quantum efficiency rises abruptly in a narrow range of $P_{\text {exc }}$ and then becomes constant. The characteristic excitation intensity corresponding to the abrupt rise in PL quantum efficiency shifts to higher values of $P_{\text {exc }}$ with increasing temperature, in agreement with Eq. (4). In the range of PL intensities that could be reliably measured and where the BL band could be distinguished from the background signal produced by other transitions, there are no significant differences between the $n$-type and $p$-type cases shown in Fig. 3 with dashed and solid lines, respectively.

\section{A. Parameters of the model}

Although there seem to be a large number of parameters in the model, some of these are known from independent experiments, and the others are constrained to be within a fairly narrow range of reasonable values. All the parameters used in the fits of the rate-equation model to the experimental data have been chosen to be consistent with all other measurements on similar samples. We begin with the ionization energies and capture coefficients for the acceptor and shallow donor, which are well characterized, and some are known from independent studies. For example, from fitting the temperature dependence of the BL band intensity in conductive $n$-type $\mathrm{GaN},{ }^{22}$ the hole-capture coefficient for the $\mathrm{Zn}_{\mathrm{Ga}}$ acceptor was estimated as $C_{p A}=7 \times 10^{-7} \mathrm{~cm}^{3} / \mathrm{s}$. From time-resolved PL experiments on conductive $n$-type $\mathrm{GaN},{ }^{23}$ the electron capture coefficient for the $\mathrm{Zn}_{\mathrm{Ga}}$ acceptor was estimated as $C_{n A}=$ $4 \times 10^{-13} \mathrm{~cm}^{3} / \mathrm{s}$. PL spectroscopy ${ }^{5}$ is used to determine the ionization energies of the shallow donor and the $\mathrm{Zn}_{\mathrm{Ga}}$ acceptor. Moreover, the exact value of the parameter $E_{D}$ is not important for the fit when $E_{D}<50 \mathrm{meV}$, while the parameter $E_{A}$ can be found quite accurately from the fit of the temperature dependencies of the PL intensity because the shift of the abrupt thermal quenching with increasing excitation intensity is very sensitive to this parameter. ${ }^{1,21}$ The fits are not sensitive to the value of the parameter $C_{n D}$ when $T>100 \mathrm{~K}$, yet from an insignificant rise of the BL intensity with decreasing temperature from 100 to $10 \mathrm{~K}$, we conclude that $C_{n D}<C_{n S}$.

Regarding the concentrations of defect species, it is known from SIMS measurements and from other experiments that the concentration of uncontrolled shallow donors is commonly of 
the order of $10^{17} \mathrm{~cm}^{-3}$ in undoped GaN. ${ }^{5}$ For $p$-type GaN, we expect that the concentration of shallow donors may be even higher because of self-compensation. The value of $N_{A}-N_{D}$ can be determined from Eq. (5), in which the parameter $B$ can be estimated from the experimentally determined values of the characteristic temperature $T^{*}$ of the abrupt PL quenching at different excitation intensities. The absolute internal quantum efficiency $\eta$ can be found by comparison of the PL intensity with that from calibrated samples. ${ }^{1}$ By using this approach, we find that $N_{A} \approx 3 \times 10^{17} \mathrm{~cm}^{-3}$ and $2 \times 10^{18} \mathrm{~cm}^{-3}$ for samples ap269 and s452, respectively. As can be seen in Table I, these values are about an order of magnitude lower than the concentrations of $\mathrm{Zn}$ atoms in these samples as estimated from SIMS measurements. However, it is possible that not all $\mathrm{Zn}$ atoms substitute for $\mathrm{Ga}$ atoms in $\mathrm{Zn}$-doped $\mathrm{GaN}$ to form radiative centers, which would lead to an overestimate of the acceptor concentration $N_{A}$.

With $N_{D}$ and $N_{A}$ known, the parameter $C_{D A}$ can be estimated from Eq. (41), where the value of $G_{2}$ can be found from the experimental dependence of the PL efficiency on excitation intensity, and this implicitly uses the step-function absorption model. When the exponential absorption model is used instead, the downturn in the $\eta(G)$ curve in region IIb shifts to higher excitation intensities, as can be seen in Fig. 10, as shown by the long-dashed curve. In order to fit the data with this exponential absorption model, as shown by the solid curve, the value of $C_{D A}$ must be reduced slightly. Alternatively, $C_{D A}$ can be roughly estimated from theory in the effective-mass approximation, according to which $C_{D A} / C_{n A} \approx\left(a_{D} / a_{A}\right)^{3}$, where $a_{D}$ and $a_{A}$ are the Bohr radii for the bound electron and hole, respectively. ${ }^{24}$ Therefore, we expect that $C_{D A} \gg C_{n A}$ in the case of the shallow donor and the $\mathrm{Zn}_{\mathrm{Ga}}$ acceptor in $\mathrm{GaN}$.

This leaves the remaining unknown parameters in the model as the concentration $N_{S}$ and the carrier capture coefficients $C_{n S}$ and $C_{p S}$ of the dominant nonradiative center. We expect that $N_{S}$ is of the same order of magnitude as $N_{A}$ and $N_{D}$ in $\mathrm{Zn}$-doped GaN samples. Since some of the GaN:Zn samples that have been studied demonstrated behavior typical of $p$ type, ${ }^{1}$ for these samples $N_{S}<N_{A}-N_{D}$. On the other hand, the product of $C_{p S}$ and $N_{S}$ can be estimated from the experimentally measured PL efficiency $\eta_{0}$ in region IIa by using Eq. (36), which can be rewritten as

$$
C_{p S} N_{S}=C_{p A} N_{D} \frac{1-\eta_{0}}{\eta_{0}} .
$$

Assuming that $N_{D} \approx 10^{17} \mathrm{~cm}^{-3}$ and $N_{S} \approx 10^{17}-10^{18} \mathrm{~cm}^{-3}$, and taking $\eta_{0}=0.2$ and $C_{p A}=7 \times 10^{-7} \mathrm{~cm}^{3} \mathrm{~s}^{-1}$, we obtain that $C_{p S} \approx 3 \times 10^{-7}$ to $3 \times 10^{-6} \mathrm{~cm}^{3} \mathrm{~s}^{-1}$. The coefficient $C_{n S}$ (or at least the product $C_{n S} N_{S}$ if $N_{S}$ is unknown) can be found from the ratio $C_{D A} / C_{n S}$, which, in turn, can be estimated from the value of the ratio $R^{*}$ characterizing the drop in PL quantum efficiency defined in Eq. (42). In Ref. 1, we concluded that it is a very small value of $\zeta$, which is given in Eq. (13), rather than a precise compensation $\left(N_{S}+N_{D} \approx N_{A}\right)$, that is responsible for the large drop (large $R^{*}$ ) because similar values of the drop were observed in several GaN:Zn samples grown in different conditions. After $\zeta$ is found from Eq. (42) using the experimentally determined $R^{*}$, the ratio $C_{D A} / C_{n S}$ can be estimated from Eq. (13), where the term containing $C_{n A}$ can be ignored for a $\mathrm{GaN}: \mathrm{Zn}$ for $T<200 \mathrm{~K}$. Typical values of $C_{n S}$ obtained in our fits are of the order of $C_{n S}=10^{-7} \mathrm{~cm}^{3} \mathrm{~s}^{-1}$, which is slightly smaller that the value of $C_{p S}$. A high value of $C_{p s}$ agrees with the fact that nonradiative recombination is usually the dominant path of carrier recombination in $\mathrm{GaN}: \mathrm{Zn}$, even at the lowest temperatures, while a relatively high value of $C_{n S}$ is consistent with an assumption that the $S$ center is a donor, and it explains why the drop of PL $R^{*}$ is very large in these samples.

We proposed earlier that the dominant nonradiative center in $\mathrm{Zn}$-doped $\mathrm{GaN}$ may be a complex defect, most probably the nitrogen vacancy-zinc complex $\left(V_{\mathrm{N}} \mathrm{Zn}_{\mathrm{Ga}}\right)$. This is a donor with very deep levels and is expected to have a low formation energy in $\mathrm{Zn}$-doped GaN. ${ }^{25}$ However, the authors calculated the holecapture coefficient $C_{p S}$ to be only $5.6 \times 10^{-10} \mathrm{~cm}^{3} \mathrm{~s}^{-1}$, a value too small to explain the high efficiency of the nonradiative recombination in this material.

To summarize, most of the parameters can be found independently, and a set of reasonable parameters used in this work and in Refs. 1 and 21 within a simple phenomenological model explains quantitatively both the temperature dependencies and the excitation intensity dependencies of the PL quantum efficiency over a wide range of both temperature and excitation intensity.

\section{B. Comparison with other experimental data}

In early work from 1939 to 1949 on PL from ZnS-CdS phosphors doped with Ag, a superlinear increase of defect-related PL intensity with excitation intensity was observed. $8,10,11$ When a segment of the $I^{P L}$ dependence with the highest slope was fit with a power-law dependence, $I^{P L} \propto\left(P_{\mathrm{exc}}\right)^{m}$, powers up to $m=3.4$ were reported. ${ }^{11}$ This is similar to some of our experimental data, where the largest power achieved was $m=4$, as shown in Fig. 2. Interestingly, in 1956, not only was an increase in PL efficiency observed for $\mathrm{Cu}$-doped $\mathrm{ZnS}$ at low excitation intensities, but also its decrease at high excitation intensities, ${ }^{26}$ very similar to our results shown in Fig. 10. The first phenomenological models were able to explain powers up to $m=2 .{ }^{15}$ To explain larger powers, empirical models were proposed. ${ }^{13}$ Later, Klasens ${ }^{17}$ suggested a model, obtained by random fits of parameters, that included two types of defects, a two-center model, and could explain the superlinear rise of PL with large $m$ observed in Ref. 11 and an unusual dependence of the PL efficiency, involving an abrupt rise at low excitation intensity and a decrease at high excitation intensity, that had been observed in Ref. 26. These are the same effects we observe and explain with our model, in which the condition of population inversion can be achieved by decreasing the temperature at fixed light excitation intensity $P_{\text {exc }}$ or by increasing light excitation intensity at fixed temperature. His model included the $A$ center, which he had as a donor with a level close to the valence band, and a nonradiative center $S$, which he had as an acceptor with a level close to the conduction band, which is clearly unphysical. He did not include the shallow donor. Since this work was done in 1958 , he did not have the advantage of a wealth of information about reasonable parameters that is available today, and so it was not apparent at the time that his parameters were unreasonable. In fact, our calculations show 
that the two-center model, when using physically reasonable parameters, fails to explain the abrupt, stepwise change in PL efficiency if the $A$ is an acceptor and the $S$ center is a donor. The inclusion of the shallow donors in the model is necessary because it allows electrons to accumulate in the conduction band and saturate the $S$ center with electrons.

\section{CONCLUSIONS}

We have observed a superlinear increase in the BL band intensity in $\mathrm{Zn}$-doped $\mathrm{GaN}$ with increasing light excitation intensity. The characteristic electron-hole-pair generation rate $G^{*}$ corresponding to the abrupt rise of the PL efficiency shifts to higher excitation intensities with increasing temperature. This phenomenon is interrelated with another unusual effect: the abrupt and tunable thermal quenching of PL observed in the same samples. ${ }^{1}$ We showed with the help of a rate-equation model that in both cases, a system involving three defect species can switch from a nearly equilibrium population of levels in the band gap at low excitation intensity (or high temperature) to a population inversion at high excitation intensity (or low temperature). This crossover is abrupt, and it can be caused by changing the temperature at fixed excitation intensity or by changing the excitation intensity at fixed temperature. A useful consequence of the rate-equation model is that it has enabled us to estimate the carrier-capture parameters of the dominant nonradiative center in $\mathrm{Zn}$-doped $\mathrm{GaN}$.

\section{ACKNOWLEDGMENTS}

The authors are grateful to A. Usikov for providing GaN samples that were made at TDI, Inc. M.F.B. and T.M. would like to thank Arthur and Ellen Bishop and Bishop Orchards for providing computational facilities.

\section{APPENDIX: DERIVATION OF APPROXIMATE ANALYTICAL SOLUTIONS TO THE MODEL FOR LOW EXCITATION INTENSITY}

At low excitation intensity, the equations can be solved approximately analytically up to first order in the electronhole-pair generation rate $G$ for both $p$-type and $n$-type semiconductors. The equations to be solved are Eqs. (7)-(12) of Ref. 1, and we repeat them here for convenience:

$$
\frac{\partial n}{\partial t}=G-C_{n S} N_{S}^{+} n-C_{n D} N_{D}^{+} n-C_{n A} N_{A}^{0} n+Q_{D} N_{D}^{0}=0,
$$

$$
\begin{gathered}
\frac{\partial p}{\partial t}=G-C_{p S} N_{S}^{0} p-C_{p A} N_{A}^{-} p+Q_{A} N_{A}^{0}=0 \\
\frac{\partial N_{D}^{0}}{\partial t}=C_{n D} N_{D}^{+} n-Q_{D} N_{D}^{0}-C_{D A} N_{D}^{0} N_{A}^{0}=0 \\
\frac{\partial N_{S}^{0}}{\partial t}=C_{n S} N_{S}^{+} n-C_{p S} N_{S}^{0} p=0 \\
\frac{\partial N_{A}^{0}}{\partial t}=C_{D A} N_{D}^{0} N_{A}^{0}+C_{n A} N_{A}^{0} n-C_{p A} N_{A}^{-} p+Q_{A} N_{A}^{0}=0
\end{gathered}
$$

$$
p+N_{S}^{+}+N_{D}^{+}=n+N_{A}^{-} .
$$

Here, in agreement with Fig. 5, Eqs. (A1) and (A2) describe the balance of electrons and holes in the conduction and valence bands, respectively; Eqs. (A3), (A4), and (A5) describe the flow of electrons and holes through shallow donors $D$, acceptors $A$, and nonradiative centers $S$, respectively. Finally, Eq. (A6) describes the conservation of charge in a semiconductor under the conditions of PL. Only five of these six equations are linearly independent.

We solve these equations by a form of perturbation theory known as successive approximations as powers of the electron-hole-pair generation rate $G$. We write each carrier concentration as the sum of a zeroth- and first-order part, where we write $G$ explicitly in the first-order term. For instance, the free-electron concentration will be written as

$$
n=n^{(0)}+G \bar{n}^{(1)}
$$

where $n^{(0)}$ is the zeroth-order part and $\bar{n}^{(1)}$ is the coefficient of $G$ in the second-order part. All the concentrations are written with this same form. For a high-resistivity semiconductor, the zeroth-order free electron concentration is zero, as is the neutral shallow donor concentration. Therefore, setting $n^{(0)}=$ $N_{D}^{0(0)}=0$ and $N_{D}^{+(0)}=N_{D}$, in zeroth order, Eq. (A1) has no zeroth-order terms. In zeroth order, writing $N_{A}^{-}=N_{A}-N_{A}^{0}$, Eq. (A2) becomes

$$
\left(C_{p S} N_{S}^{0(0)}+C_{p A} N_{A}-C_{p A} N_{A}^{0(0)}\right) p^{(0)}=Q_{A} N_{A}^{0(0)}
$$

The zeroth-order version of Eq. (A3), with $n^{(0)}=N_{D}^{0(0)}=0$, yields no zeroth-order terms. If we make the same substitution into Eq. (A4), we get the particularly simple expression,

$$
-C_{p S} N_{S}^{0(0)} p^{(0)}=0 .
$$

For Eq. (A5), substituting $N_{A}^{-}=N_{A}-N_{A}^{0}$ and $n^{(0)}=$ $N_{D}^{0(0)}=0$, the zeroth-order equation becomes

$$
C_{p A}\left(N_{A}-N_{A}^{0(0)}\right) p^{(0)}=Q_{A} N_{A}^{0(0)} .
$$

In the charge conservation equation, when we set $N_{A}^{-}=N_{A}-$ $N_{A}^{0}, N_{D}^{+}=N_{D}-N_{D}^{0}, N_{S}^{+}=N_{S}-N_{S}^{0}$, and $n^{(0)}=N_{D}^{0(0)}=0$, Eq. (A6) becomes

$$
-N_{S}^{0(0)}+N_{A}^{0(0)}+p^{(0)}=N_{A}-N_{D}-N_{S} .
$$

We therefore have only four of six remaining equations, and only three are linearly independent. We first notice that Eq. (A9) requires that either $N_{S}^{(0)}=0$ or $p^{(0)}=0$. From Eq. (A11), we see that $N_{S}^{0(0)}=0$ is possible only if $N_{A}-$ $N_{D}-N_{S}>0$, which corresponds to a $p$-type semiconductor. From the same equation, we see that if $p^{(0)}=0$, then from Eq. (A8), we obtain $N_{A}^{0(0)}=0$, and this is possible in Eq. (A11) only if $N_{A}-N_{D}-N_{S}<0$, which corresponds to an $n$-type semiconductor. Therefore, we must consider separately the cases of $p$ type and $n$ type.

We start with $p$ type, where $N_{S}^{0(0)}=0$, and we use Eq. (A11) to solve for $N_{A}^{(0)}$ in terms of $p^{(0)}$ and substitute the result into Eq. (A10) to obtain a quadratic equation in $p^{(0)}$, given by

$$
C_{p A}\left(N_{D}+N_{S}+p^{(0)}\right) p^{(0)}=Q_{A}\left(N_{A}-N_{D}-N_{S}-p^{(0)}\right) .
$$


The solution to this quadratic that gives a positive concentration is

$$
\begin{aligned}
p^{(0)}= & -\frac{1}{2}\left(N_{D}+N_{S}+\frac{Q_{A}}{C_{p A}}\right) \\
& +\frac{1}{2} \sqrt{\left(N_{D}+N_{S}+\frac{Q_{A}}{C_{p A}}\right)^{2}+4 \frac{Q_{A}}{C_{p A}}\left(N_{A}-N_{D}-N_{S}\right) .}
\end{aligned}
$$

If $4 \frac{Q_{A}}{C_{p A}}\left(N_{A}-N_{D}-N_{S}\right) \ll\left(N_{D}+N_{S}+\frac{Q_{A}}{C_{p A}}\right)^{2}$, which is generally true for our high-resistivity $\mathrm{Zn}$-doped $\mathrm{GaN}$ for $T>100$ $\mathrm{K}$, then we can expand the square root and obtain the simple result

$$
p^{(0)} \approx \frac{Q_{A}}{C_{p A}} \frac{\left(N_{A}-N_{D}-N_{S}\right)}{\left(N_{D}+N_{S}\right)} .
$$

This is the same as the result given in Eq. (23) for a $p$-type semiconductor. Then $N_{A}^{0(0)}=N_{A}-N_{D}-N_{S}-p^{(0)}$. For $n$ type, setting $p^{(0)}=N_{A}^{0(0)}=0$, we can solve Eq. (A11) to obtain the zeroth-order solution,

$$
N_{S}^{0(0)}=N_{D}+N_{S}-N_{A} .
$$

These zeroth-order solutions can now be substituted into the first-order equations derived from Eqs. (A1)-(A6). We begin with the form of the equations that is valid for both $p$ type and $n$ type, where $n^{(0)}=N_{D}^{0(0)}=0$. Recalling that a first-order concentration with a bar over it means that it has been divided by $G$, the first-order version of Eq. (A1), which has been divided through by $G$, is

$$
\begin{aligned}
& \left(C_{n S} N_{S}+C_{n D} N_{D}-C_{n S} N_{S}^{0(0)}+C_{n A} N_{A}^{0(0)}\right) \bar{n}^{(1)} \\
& \quad=1+Q_{D} \bar{N}_{D}^{0(1)} .
\end{aligned}
$$

To obtain the first-order version of Eq. (A2), we substitute $N_{A}^{-}=N_{A}-N_{A}^{0}$ and divide through by $G$ to obtain

$$
\begin{aligned}
& {\left[C_{p S} N_{S}^{0(0)}+C_{p A}\left(N_{A}-N_{A}^{0(0)}\right)\right] \bar{p}^{(1)}} \\
& \quad=1-C_{p S} p^{(0)} \bar{N}_{S}^{0(1)}+\left(C_{p A} p^{(0)}+Q_{A}\right) \bar{N}_{A}^{0(1)} .
\end{aligned}
$$

For Eq. (A3), we substitute $N_{D}^{+}=N_{D}$ and get in first order

$$
C_{n D} N_{D} \bar{n}^{(1)}=\left(C_{D A} N_{A}^{0(0)}+Q_{D}\right) \bar{N}_{D}^{0(1)} .
$$

Substituting $N_{S}^{+}=N_{S}-N_{S}^{0}$ into Eq. (A4) we find the firstorder equation

$$
C_{n S}\left(N_{S}-N_{S}^{0(0)}\right) \bar{n}^{(1)}=C_{p S} N_{S}^{0(0)} \bar{p}^{(1)}+C_{p S} p^{(0)} \bar{N}_{S}^{0(1)} .
$$

Obtaining the first-order version of Eq. (A5) requires setting $N_{A}^{-}=N_{A}-N_{A}^{0}$, and we have to first order

$$
\begin{aligned}
& C_{D A} N_{A}^{0(0)} \bar{N}_{D}^{0(1)}+C_{n A} N_{A}^{0(0)} \bar{n}^{(1)} \\
& \quad=C_{p A}\left(N_{A}-N_{A}^{0(0)}\right) \bar{p}^{(1)}-\left(C_{p A} p^{(0)}+Q_{A}\right) \bar{N}_{A}^{0(1)} .
\end{aligned}
$$

Finally, in the charge conservation equation (A6), we substitute $N_{A}^{-}=N_{A}-N_{A}^{0}, N_{D}^{+}=N_{D}-N_{D}^{0}, N_{S}^{+}=N_{S}-N_{S}^{0}$, and we have in first order

$$
\bar{p}^{(1)}-\bar{N}_{S}^{0(1)}-\bar{N}_{D}^{0(1)}=\bar{n}^{(1)}-\bar{N}_{A}^{0(1)} .
$$

In order to proceed, we need to consider $p$ type and $n$ type separately. For $p$ type, we have in zeroth order $n^{(0)}=N_{D}^{(0)}=$ $N_{S}^{0(0)}=0, N_{A}^{0(0)}=N_{A}-N_{D}-N_{S}-p^{(0)}$, with $p^{(0)}$ given by Eq. (A13) or approximately by Eq. (A14). From this point forward, we use the approximate version. We begin with Eq. (A19) to get $\bar{N}_{S}^{0(1)}$ in terms of $\bar{n}^{(1)}$ as

$$
\bar{N}_{S}^{O(1)}=\frac{C_{n S} N_{S}}{C_{p S} p^{(0)}} \bar{n}^{(1)} .
$$

We next solve Eq. (A18) for $\bar{N}_{D}^{0(1)}$ in terms of $\bar{n}^{(1)}$ as

$$
\bar{N}_{D}^{0(1)}=\frac{C_{n D} N_{D} \bar{n}^{(1)}}{C_{D A}\left(N_{A}-N_{D}-N_{S}-p^{(0)}\right)+Q_{D}} .
$$

We now substitute the expression $\bar{N}_{D}^{0(1)}$ from Eq. (A23) into Eq. (A16) and solve for $\bar{n}^{(1)}$, with the result

$$
\begin{aligned}
\bar{n}^{(1)}= & \left\{\left(C_{n S} N_{S}+C_{n D} N_{D}\right)+C_{n A}\left(N_{A}-N_{D}-N_{S}-p^{(0)}\right)\right. \\
& \left.-Q_{D} \frac{C_{n D} N_{D}}{\left[C_{D A}\left(N_{A}-N_{D}-N_{S}-p^{(0)}\right)+Q_{D}\right]}\right\}^{-1} .
\end{aligned}
$$

For the GaN high-resistivity Zn-doped case discussed here, $C_{n A}\left(N_{A}-N_{D}-N_{S}-p^{(0)}\right)$ can be neglected compared with $\left(C_{n S} N_{S}+C_{n D} N_{D}\right)$, and $C_{D A}\left(N_{A}-N_{D}-N_{S}-p^{(0)}\right)$ can be neglected compared with $Q_{D}$. Therefore, $\bar{n}^{(1)}$ reduces to

$$
\bar{n}^{(1)}=\frac{1}{C_{n S} N_{S}},
$$

$\bar{N}_{D}^{0(1)}$ reduces to

$$
\bar{N}_{D}^{0(1)}=\frac{C_{n D} N_{D}}{C_{n S} N_{S} Q_{D}},
$$

and $\bar{N}_{S}^{0(1)}$ reduces to

$$
\bar{N}_{S}^{0(1)}=\frac{1}{C_{p S} p^{(0)}} .
$$

Having $\bar{n}^{(1)}$, we can solve for $\bar{N}_{A}^{0(1)}$ in terms of $\bar{n}^{(1)}, \bar{p}^{(1)}, \bar{N}_{D}^{0(1)}$, and $\bar{N}_{S}^{0(1)}$ from Eq. (A21) and substitute into Eq. (A17). We then substitute for $\bar{N}_{S}^{0(1)}$ and $\bar{N}_{D}^{0(1)}$ from Eqs. (A26) and (A27), respectively, and we obtain an equation in terms of $\bar{n}^{(1)}$ and $\bar{p}^{(1)}$. We then substitute for $\bar{n}^{(1)}$ from Eq. (A25). In addition, we neglect $C_{n D} N_{D} / Q_{D}$ compared with unity. Finally, we substitute the approximate solution for $p^{(0)}$ from Eq. (A14). The resulting solution for $\bar{p}^{(1)}$ is

$$
\begin{aligned}
\bar{p}^{(1)}= & \frac{N_{A}}{C_{p S}\left(N_{D}+N_{S}\right)\left(N_{A}-N_{D}-N_{S}\right)} \\
& \times \frac{\left[1+\frac{Q_{A}}{C_{n S} N_{S}} \frac{C_{p S}}{C_{p A}} \frac{\left(N_{A}-N_{D}-N_{S}\right)}{\left(N_{D}+N_{S}\right)}\right]}{\left[1+\frac{Q_{A}}{C_{p A}\left(N_{D}+N_{S}\right)^{2}}\left(2 N_{A}-N_{D}-N_{S}\right)\right]} .
\end{aligned}
$$

For the parameters used in this paper, the terms involving $Q_{A}$ are small compared with unity and may therefore be neglected. Therefore, we have the simple expression

$$
\bar{p}^{(1)} \approx \frac{N_{A}}{C_{p S}\left(N_{D}+N_{S}\right)\left(N_{A}-N_{D}-N_{S}\right)} .
$$


The free hole concentration for $p$ type, up to and including first order, is then

$$
p=\frac{Q_{A}}{C_{p A}} \frac{\left(N_{A}-N_{D}-N_{S}\right)}{\left(N_{D}+N_{S}\right)}+G \bar{p}^{(1)},
$$

where $\bar{p}^{(1)}$ is given by Eq. (A29). To find $N_{A}^{0}$, we use Eq. (A21) together with the expressions for $\bar{n}^{(1)}, \bar{N}_{D}^{0(1)}, \bar{N}_{S}^{0(1)}$, and $\bar{p}^{(1)}$ from Eqs. (A25), (A26), (A27), and (A29), respectively, and neglect $C_{n D} N_{D} / Q_{D}$ compared with unity. Therefore, up to and including first order $N_{A}^{0}$ is given by

$$
\begin{aligned}
N_{A}^{0}= & N_{A}-N_{D}-N_{S}-p^{(0)} \\
& -G\left(\bar{p}^{(1)}+\bar{N}_{S}^{0(1)}+\bar{n}^{(1)}+\bar{N}_{D}^{0(1)}\right) \\
= & N_{A}-N_{D}-N_{S}-\frac{Q_{A}}{C_{p A}} \frac{\left(N_{A}-N_{D}-N_{S}\right)}{\left(N_{D}+N_{S}\right)} \\
& +\frac{C_{p A}}{C_{p S}} \frac{G}{Q_{A}} \frac{\left(N_{D}+N_{S}\right)}{\left(N_{A}-N_{D}-N_{S}\right)} \\
& \times\left[1+\frac{Q_{A} N_{A}}{C_{p A}\left(N_{D}+N_{S}\right)^{2}}\right. \\
& \left.+\frac{C_{p S}}{C_{p A}} \frac{Q_{A}}{C_{n S} N_{S}} \frac{\left(N_{A}-N_{D}-N_{S}\right)}{\left(N_{D}+N_{S}\right)}\right] .
\end{aligned}
$$

Using $N_{A}^{0}$ from Eq. (A31) and neglecting the terms in square brackets containing $Q_{A}$, since they are small compared with one, and using $N_{D}^{0}=G \bar{N}_{D}^{0(1)}$ from Eq. (A26) and $n=G \bar{n}^{(1)}$ from Eq. (A25), the quantum efficiency is given for $p$ type from Eqs. (3) and (16) by

$$
\begin{aligned}
\eta & =\frac{1}{G}\left(C_{D A} N_{D}^{0}+C_{n A} n\right) N_{A}^{0} \\
& =\zeta \frac{\left(N_{A}-N_{D}-N_{S}\right)}{N_{S}}\left[1-\frac{Q_{A}}{C_{p A}\left(N_{D}+N_{S}\right)}\right],
\end{aligned}
$$

where $\zeta$ is given in Eq. (13). We have omitted the term in $N_{A}^{0}$ that is proportional to $G$, because such a term is actually second order in $G$ in the PL intensity. In order to include this term, we would need to calculate $n$ and $N_{D}^{0}$ up to second order in $G$ also. The quantum efficiency given by Eq. (A32) is the same expression for the $p$-type quantum efficiency in region Ia, as given in Eq. (28), if we neglect the term containing $Q_{A}$.

The solution of the equations to first order is much simpler for the $n$-type case. In that case, we substitute $p^{(0)}=N_{A}^{0(0)}=0$ and $N_{S}^{0(0)}=N_{D}+N_{S}-N_{A}$ into Eqs. (A16)-(A21). We first use Eq. (A18) to solve for $\bar{N}_{D}^{0(1)}$ in terms of $n^{(1)}$ as

$$
\bar{N}_{D}^{0(1)}=\frac{C_{n D} N_{D}}{Q_{D}} \bar{n}^{(1)} .
$$

From Eq. (A20), we solve for $\bar{N}_{A}^{0(1)}$ in terms of $\bar{p}^{(1)}$ as

$$
\bar{N}_{A}^{(1)}=\frac{C_{p A} N_{A}}{Q_{A}} \bar{p}^{(1)} .
$$

We can substitute this into Eq. (A17) to get $\bar{p}^{(1)}$, and from this get $p$ to first order, which is

$$
p=G \bar{p}^{(1)}=\frac{G}{C_{p S}\left(N_{D}+N_{S}-N_{A}\right)} .
$$

Then we can get $\bar{N}_{A}^{0(1)}$ and $N_{A}^{0}$ to first order,

$$
N_{A}^{0}=G \bar{N}_{A}^{0(1)}=\frac{C_{p A}}{C_{p S}} \frac{G N_{A}}{Q_{A}\left(N_{D}+N_{S}-N_{A}\right)} .
$$

From Eq. (A19), we can get $\bar{n}^{(1)}$ in terms of $\bar{p}^{(1)}$ and then substitute from Eq. (A35) to produce $n$ to first order

$$
n=G \bar{n}^{(1)}=\frac{G}{C_{n S}\left(N_{A}-N_{D}\right)} .
$$

This allows us to write $\bar{N}_{D}^{0(1)}$, and thus $N_{D}^{0}$, from Eq. (A33) as

$$
N_{D}^{0}=G \bar{N}_{D}^{0(1)}=\frac{C_{n D} N_{D}}{Q_{D}} \frac{G}{C_{n S}\left(N_{A}-N_{D}\right)} .
$$

We can therefore calculate the quantum efficiency, and substituting $\zeta$ from Eq. (13) and $N_{2}$ from Eq. (12), we have for the quantum efficiency for $n$ type,

$$
\eta=\frac{\zeta N_{2} N_{A}}{\left(N_{A}-N_{D}\right)\left(N_{D}+N_{S}-N_{A}\right)} .
$$

This is the same form given in Eq. (29) for $n$ type in region Ia. *mreshchi@vcu.edu

${ }^{1}$ M. A. Reshchikov, A. A. Kvasov, M. F. Bishop, T. McMullen, A. Usikov, V. Soukhoveev, and V. A. Dmitriev, Phys. Rev. B 84, 075212 (2011).

${ }^{2}$ D. Curie, Luminescence in Crystals (Wiley \& Sons, New York, 1963).

${ }^{3}$ H. B. Bebb and E. W. Williams, in Semiconductors and Semimetals, edited by R. K. Willardson and A. C. Beer, Vol. 8 (Academic Press, New York, London, 1972), pp. 181-420.

${ }^{4}$ J. I. Pankove, Optical Processes in Semiconductors (Dover, New York, 1971).

${ }^{5}$ M. A. Reshchikov and H. Morkoç, J. Appl. Phys. 97, 061301 (2005).

${ }^{6}$ K. D. Glinchuk, A. V. Prokhorovich, and V. E. Ronionov, Sov. Phys. Semicond. 11, 18 (1977).
${ }^{7}$ T. Schmidt, K. Lischka, and W. Zulehner, Phys. Rev. B 45, 8989 (1992).

${ }^{8}$ N. Riehl, Z. Tech. Phys. 20, 152 (1939).

${ }^{9}$ J. H. Gisolf and F. A. Kröger, Physica 6, 1101 (1939).

${ }^{10}$ F. Urbach, A. Urbach, and M. Schwartz, J. Opt. Soc. Am. 37, 122 (1947).

${ }^{11}$ N. R. Nail, F. Urbach, and D. Pearlman, J. Opt. Soc. Am. 39, 690 (1949).

${ }^{12}$ F. I. Vergunas and F. F. Gavrilov, Zh. Eksp. Teor. Fiz. 18, 873 (1948).

${ }^{13}$ G. F. J. Garlick and A. F. Gibson, J. Opt. Soc. Am. 39, 935 (1949).

${ }^{14}$ M. N. Alentsev, V. V. Antonov-Romanovsky, and L. A. Vinokurov, Dokl. Akad. Nauk SSSR 96, 1133 (1954).

${ }^{15}$ M. E. Wise and H. A. Klasens, J. Opt. Soc. Am. 38, 226 (1948).

${ }^{16}$ S. Roberts and F. E. Williams, J. Opt. Soc. Am. 40, 516 (1950). 
${ }^{17}$ H. A. Klasens, J. Phys. Chem. Solids 7, 175 (1958).

${ }^{18}$ C. A. Duboc, Br. J. Appl. Phys. 6, S107 (1955).

${ }^{19} \mathrm{~S}$. Komiya, A. Yamaguchi, I. Umebu, and T. Kotani, Jpn. J. Appl. Phys. 23, 308 (1984).

${ }^{20}$ S. Komiya, A. Yamaguchi, and I. Umebu, Solid-State Electron. 29, 235 (1986).

${ }^{21}$ M. A. Reshchikov, Phys. Rev. B 85, 245203 (2012).
${ }^{22}$ M. A. Reshchikov, M. Foussekis, J. D. McNamara, A. Behrends, A. Bakin, and A. Waag, J. Appl. Phys. 111, 073106 (2012).

${ }^{23}$ R. Y. Korotkov, M. A. Reshchikov, and B. W. Wessels, Physica B 325, 1 (2003).

${ }^{24}$ A. P. Levanyuk and V. V. Osipov, Sov. Phys. Usp. 24, 187 (1981).

${ }^{25}$ L. Shi and L.-W. Wang, Phys. Rev. Lett. 109, 245501 (2012).

${ }^{26}$ V. V. Antonov-Romanovsky, J. Phys. Radium 17, 694 (1956). 